\title{
1 Accuracy and precision of dolphin group size estimates
}

4 Tim Gerrodette ${ }^{1, *}$, Wayne L. Perryman ${ }^{1}$, Cornelia S. Oedekoven ${ }^{2}$

$6{ }^{1}$ NOAA Fisheries, Southwest Fisheries Science Center, 8901 La Jolla Shores Dr., La Jolla,

7 California 92037, USA

$8 \quad{ }^{2}$ Centre for Research into Ecological and Environmental Modelling, The Observatory, Buchanan

9 Gardens, University of St. Andrews, St. Andrews, Fife KY169LZ, Scotland, UK

$10{ }^{*}$ Corresponding author: tim.gerrodette@noaa.gov 
12 Abstract: Estimating the number of dolphins in a group is a challenging task. To assess the

13 accuracy and precision of dolphin group size estimates, observer estimates were compared to

14 counts from large-format vertical aerial photographs. During 11 research cruises, a total of 2,435

15 size estimates of 434 groups were made by 59 observers. Observer estimates were modeled as a

16 function of the photo count in a hierarchical Bayesian framework. Accuracy varied widely

17 among observers, and somewhat less widely among dolphin species. Most observers tended to

18 underestimate, and the tendency increased with group size. Groups of 25, 50, 100, and 500 were

19 underestimated by $<1 \%, 16 \%, 27 \%$, and $47 \%$, respectively, on average. Precision of group size

20 estimates was low, and estimates were highly variable among observers for the same group.

21 Predicted true group size, given an observer estimate, was larger than the observer estimate for

22 groups of more than about 25 dolphins. Predicted group size had low precision, with coefficients

23 of variation ranging from 0.7 to 1.9 . Studies which depend on group size estimates will be

24 improved if the tendency to underestimate group size and the high uncertainty of group size

25 estimates are included in the analysis.

28 Keywords: group size estimation, abundance estimation, aerial photography, Bayesian

29 hierarchical model, random-effects model, reverse jump MCMC 
Estimation of group size is an important component of ecological and behavioral studies

33 of animals which occur in groups. However, estimation of group size in wildlife studies can be

34 difficult. Replicate counts of birds showed high variation (Ryan and Cooper 1989), the number

35 of birds was undercounted in aerial surveys (Bayliss and Yeomans 1990), and known group sizes

36 of elk were underestimated from a helicopter (Cogan and Diefenbach 1998). Even counting the

37 number of birds in photographs had a negative bias (Erwin 1982). Experiments in visual

38 perception have shown a tendency to underestimate the size of large groups of objects (Krueger

39 1972), apparently related to distortions produced by saccadic (“jerky”) eye movements (Binda et

40 al. 2011). Determining the size of a group of cetaceans is particularly challenging because of

41 several characteristics that make group size estimation difficult: (1) the animals are moving; (2)

42 an unknown fraction of the group is underwater at any moment; (3) the fraction underwater

43 changes with behavior; (4) groups can be large; and (5) the distribution of group sizes is usually

44 skewed, with a few groups much larger than the mean.

Accurate estimation of group size is necessary for unbiased estimation of abundance. In

46 standard distance sampling (e.g., line transects), the density of groups is estimated and then

47 multiplied by an estimate of expected group size (Buckland et al. 2001). Alternatively, group

48 size may be a covariate of the detection process and expected group size is not estimated

49 explicitly (Borchers and Burnham 2004). In either case it is assumed that group sizes are

50 measured accurately. Using earlier subsets of the photographic calibration data presented here,

51 some line-transect analyses have used group size estimates corrected by observer-specific

52 calibration factors (Gerrodette and Forcada 2005, Barlow and Forney 2007). In most studies,

53 however, correction factors for group size estimation are not available. 
Assessing precision of group size estimates is equally important. Even if group sizes

55 were to be estimated accurately on average, there is measurement error associated with each

56 group size estimate. Including the variability associated with group size estimates is necessary

57 for proper assessment of uncertainty. If measurement error is not included, variance of estimates

58 of abundance and other quantities that depend on group size estimates will be too small. In other

59 words, an important source of uncertainty will not be included in the analysis, and conclusions

60 may appear to be more precise than they should be.

61 In this large field study, we measured the accuracy and precision of dolphin group size

62 estimates. True group size was assessed with counts from high-quality vertical aerial

63 photographs, and ship-based observer estimates were calibrated against these counts. The

64 tendencies of different individual observers to under- or over-estimate group size were estimated

65 in a hierarchical Bayesian framework, for different group sizes, species, and sea-state conditions.

66 The performance of a new (out-of-sample) observer was predicted by integrating over observer

67 and/or species effects. Given an observer estimate, we inferred true group size by sampling

68 posterior distributions.

Methods

Field methods

Photographs of dolphin groups were collected during 11 research cruises between 1987

73 and 2006 in the eastern tropical Pacific Ocean. During all cruises except the last, the NOAA

74 vessel David Starr Jordan carried a Hughes 500D helicopter equipped with two large-format

75 military reconnaissance cameras mounted below the fuselage. During the 2006 cruise, images 
were collected with the same camera systems mounted in a NOAA Twin Otter fixed wing

77 aircraft. Under conditions of sun angle (generally mid-morning and mid-afternoon) and sea state

78 (generally Beaufort 0-4) that allowed dolphins to be clearly visible from above, vertical

79 photographs of dolphin groups were taken from an altitude of 200-300m (Gilpatrick 1993). The

80 camera recorded images on $114 \mathrm{~mm}$ negatives, and had a motion-compensation system that

81 moved the film at the same speed that the image was moving within the camera, thus eliminating

82 blurring due to the forward motion of the aircraft. The cycle rate of the camera was adjusted to

83 achieve $80 \%$ overlap between adjacent frames during a photographic pass over a dolphin group.

84 The number of photographic passes of each dolphin group varied with group size, configuration 85 and behavior.

After a group of dolphins had been photographed, the group was approached by the ship

87 in a way to give the marine mammal observers on the ship the best possible view of the whole

88 group, considering wind, swell, and sun angle. All observers who had adequate views of the

89 group, usually all six observers on the ship, made their best estimates of group size. We refer to

90 these estimates as the "observer estimates." Observers usually first detected dolphins with $25 \mathrm{X}$

91 binoculars, but switched to 7X binoculars and then to naked eye as the ship approached the

92 group. The minimum approach distance varied with group size and behavior, but typically was

93 10-50m. Observers made group size estimates independently and did not discuss their estimates

94 with each other, either during the sighting or afterward. Independence in this context refers to

95 the behavioral independence of the observers, not to the statistical independence of their

96 estimates. All observers had previous experience in cetacean field work. Before each cruise,

97 observers were given training on group size estimation, including tests with known numbers of 
98 static objects, computer simulations of moving, intermittently visible objects, and instruction on

99 counting by subgroups (e.g., by tens or fifties) for more consistent estimation.

100

\section{Laboratory methods}

The aerial photographs of dolphin groups were reviewed on light tables equipped with dissection microscopes (Gilpatrick 1993). Photographs were compared with notes recorded during the photographic passes to ensure that the entire group was captured within the series of images that made up a photograph pass. For groups that were successfully photographed, the best pass was selected, and three readers independently counted the number of dolphins in the group from the series of images. If the $\mathrm{CV}$ among counts was $>0.1$, or if notes by aerial and shipboard observers indicated that there was confusion over the identity of the group, the group was not included in the data analyzed here (Gilpatrick 1993).

To qualify as a "calibration school" for this analysis, the whole group had to be photographed from the air with a series of overlapping photographs, the photo counts of the three independent readers had to agree closely, and the shipboard observers had to view the whole group for a sufficient time to make good estimates. Calibration schools were thus not a random sample of all dolphin groups, but rather a selected set for which we were confident that true group size could be accurately determined. We omitted as outliers eight cases for which there was a large (greater than a factor of four) discrepancy between mean photo count and mean observer estimate, probably a result of undetected splitting or coalescence of groups after photography but before observer estimates. A total of 434 groups met these criteria as calibration schools, with 2,435 estimates of group size by 59 observers.

\section{Statistical model}


121 photograph readers for each calibration school, and refer to this as the "photo count." This

122 measure of true group size had some error (variation among the three readers), but this variation

123 (mean photo count $\mathrm{CV}$ over all groups $=0.047$ ) was much smaller than the variation among

124 observer estimates of the same groups (mean $\mathrm{CV}=0.42$ ). Preliminary exploration of the data

125 suggested that, on a log-log scale, observer estimates could be linearly related to photo counts

126 and that variance was approximately constant over a large range of group sizes (Fig. 1A). In

127 addition, observers varied widely in the accuracy of their group size estimates (Fig. 1B). We

128 evaluated a variety of linear and nonlinear models in a frequentist setting, with both fixed and

129 random effects, with $\mathrm{R}$ function lmer, and used likelihood ratio tests, information criteria such as

130 AIC and DIC, and visual examinations of residual and q-q plots to identify a reasonable set of

131 candidate models. We found that dolphin species and Beaufort sea state could possibly affect the

132 accuracy of group size estimates, and that a linear model of the logarithm of photo counts

133 provided a more parsimonious fit to the data than a quadratic model.

134 Let $y_{i j}$ be the observer estimate of the size of group $i$ by observer $j$, and let $x_{i}$ be the photo

135 count of group $i$. We modeled differences among observers as random effects, and dolphin

136 species and wind conditions as fixed additional effects that might affect group size estimates.

137 The full hierarchical model may be written as

$$
\begin{aligned}
& \log \left(y_{i j}\right)=\alpha_{0 j}+\left(\alpha_{1 j}+\beta_{1}\right) \log \left(x_{i}\right)+\sum_{k=2}^{7} \beta_{k} S_{i k}+\beta_{8} B_{i}+\varepsilon_{i j} \\
& \left(\begin{array}{l}
\alpha_{0 j} \\
\alpha_{1 j}
\end{array}\right) \sim N\left(\left(\begin{array}{l}
0 \\
0
\end{array}\right),\left(\begin{array}{cc}
\sigma_{\alpha 0}^{2} & \rho \sigma_{\alpha 0} \sigma_{\alpha 1} \\
\rho \sigma_{\alpha 0} \sigma_{\alpha 1} & \sigma_{\alpha 1}^{2}
\end{array}\right)\right) \\
& \varepsilon_{i j} \sim N\left(0, \sigma_{\varepsilon}^{2}\right),
\end{aligned}
$$


139 where $\beta_{1}$ was the coefficient associated with the log of the photo counts, $\beta_{k}$ were coefficients

140 associated with six species $S_{i k}, k=2, \ldots 7$, and $\beta_{8}$ was the coefficient associated with Beaufort sea

141 state $B_{i}$. Two random effects, $\alpha_{0 j}$ and $\alpha_{1 j}$, allowed the relationship between $\log \left(y_{i j}\right)$ and $\log \left(x_{i}\right)$

142 to vary among observers, $\alpha_{0 j}$ in terms of the intercept and $\alpha_{1 j}$ in terms of the slope coefficient

$143 \beta_{1}$. The two sets of random-effects coefficients had means of zero, variances $\sigma_{\alpha 0}^{2}$ and $\sigma_{\alpha 1}^{2}$, and

144 correlation $\rho$. The assumption was that the 59 observers were a random selection from a larger

145 pool of possible observers whose group size estimation tendencies were normally distributed.

Species $S_{i k}$ entered the model as an indicator variable, with a value of 1 if group $i$ was

147 species $k$ and 0 otherwise. Species were recorded in the field at the lowest possible taxonomic

148 level, including subspecies. We combined the field identifications into six species categories:

149 pantropical spotted dolphins (Stenella attenuata, 51 groups), spinner dolphins (S. longirostris, 40

150 groups), mixed spotted-spinner dolphin groups (78 groups), striped dolphins (S. coeruleoalba,

151114 groups), common dolphins (Delphinus delphis and D. capensis, 87 groups) and other (64

152 groups). "Other” was a heterogeneous category including Risso's dolphins (Grampus griseus),

153 common bottlenose dolphins (Tursiops truncatus), rough-toothed dolphins (Steno bredanensis),

154 short-finned pilot whales (Globicephalus macrorhynchus), and other groups which did not fit

155 into the previous categories, such as mixed common-striped dolphin groups. In the eastern

156 tropical Pacific Ocean, mixed spotted-spinner dolphin groups are common, so we included these

157 as a distinct category. Sea state $B$ was recorded on the Beaufort scale as an integer from 0 to 5;

158 however, only one of the 434 calibration schools occurred in Beaufort 5 conditions, so the

159 effective range of the model was Beaufort 0-4. Because the Beaufort scale is ordered, we

160 modeled sea state as a continuous variable with a single linear coefficient. Models with sea state 
161 as a categorical variable are addressed in the Discussion. $S_{i}$ and $B_{i}$ were the same for all

162 observers for a given group $i$, so we omitted subscript $j$ for these covariates in Eq. 1.

163 We considered four variants of Eq. 1 as candidate models: model 1, without species or

164 sea-state effects $\left(\beta_{k}=0\right.$ and $\left.\beta_{8}=0\right)$; model 2, with species but without sea-state effects $\left(\beta_{8}=0\right)$;

165 model 3, with sea-state but without species effects $\left(\beta_{k}=0\right)$; and model 4 , the full model with both

166 species and sea-state effects. All four models included observers as a random effect.

167 Bayesian inference

168 To include model selection in a Bayesian framework, we fitted the models in $\mathrm{R}$ using

169 reversible jump Markov Chain Monte Carlo (RJMCMC) methods (King et al. 2009, Oedekoven

170 et al. 2014). In this approach, the model itself was treated as an additional parameter to be

171 estimated, and the joint posterior distribution included both parameters and models (Appendix

172 1). A uniform discrete prior was specified for the four models, and uniform continuous priors

173 were specified for all coefficients $\beta$ and standard deviations $\sigma$ in Eq. 1. Model probabilities

174 were calculated as the fraction of iterations of the RJMCMC chain in each model after burn-in 175 (Appendix 1).

The four models were also fitted in the BUGS language (Lunn et al. 2000) and compared

177 with the Watanabe-Akaike (or Widely Applicable) Information Criterion (WAIC) (Watanabe

178 2010). WAIC can be viewed as an improvement to the Deviance Information Criterion (DIC)

179 (Spiegelhalter et al. 2002), which has some shortcomings for hierarchical models (Plummer

180 2008, Millar 2009, Lunn et al. 2013). WAIC was calculated using pointwise predictive density

181 at the observer level from the MCMC posterior samples for each model (Gelman et al. 2014,

182 Vehtari et al. 2016). We used standard procedures to assess burn-in, autocorrelation, and 
183 convergence of the MCMC samples (Appendix 2). BUGS code is given in Appendix 3 and R 184 code for the RJMCMC analysis in Appendix 4.

185 For prediction, we sampled from the BUGS posterior samples of model 2, which had the 186 most support (see Results). We made two kinds of predictions: an observer estimate given true 187 group size and true group size given an observer estimate. For each, we predicted conditionally 188 and unconditionally on both observer and species. A conditional prediction for an observer or 189 species meant a prediction given that it was made by a particular observer or given that it was 190 made of a group of a particular species. An unconditional prediction was calculated to infer

191 estimation tendencies for a new (out-of-sample) observer and/or species - that is, estimation

192 tendencies integrated over observers or species effects. Unconditional predictions were 193 approximated by sampling observers and/or species randomly. We sampled the MCMC chain 19450,000 times with replacement, each time also randomly sampling an observer and a species for 195 unconditional inference. For the model error term $\sigma_{\varepsilon}^{2}$, we made random draws from normal 196 distributions with the MCMC sample variances. To preserve the covariance structure, we used

197 the whole set of parameter values for each selected MCMC iteration, and computed observer 198 estimate $y$ given group size, or group size $x$ given observer estimate, based on Eq. 1. We 199 checked the accuracy of our predictions by comparing them to the photo counts (Appendix 5). 200 For each of the 2,435 observer estimates, we determined if the central $95 \%$ credibility interval of 201 predicted size included the photo count.

\section{Calibration schools}


206 calibration schools represented about $8 \%$ of dolphin groups of the same species detected during

207 the 11 surveys. On average, calibration schools were larger in size (because we did not

208 photograph groups containing only a few dolphins) and were photographed in lower Beaufort sea

209 states (because it was harder to obtain clear images in windy conditions) than for all dolphin

210 groups. Importantly, the variation among independent observer estimates for a dolphin group

211 was similar for calibration schools (mean CV 0.42, interquartile range 0.29-0.50) and all detected

212 groups (mean CV 0.39, interquartile range 0.24-0.51). The number of calibration schools per

213 observer ranged from 6 to 159, with a median of 33 and a mean of 41.3.

\section{Observer estimates of dolphin group size}

215 The raw data indicated that observers generally tended to underestimate dolphin group

216 size; $69 \%$ of observer estimates were less than the photo count (Fig. 1). Both model selection

217 methods indicated that the accuracy of observer estimates was affected by the species of the

218 group but less so by Beaufort sea state. Posterior model probabilities indicated by the RJMCMC

219 chain were $0.0,0.984,0.0$, and 0.016 for models 1-4, respectively (Fig. 2). With proper selection

220 of proposal distributions, models 2 and 4 had stationary distributions throughout the history of

221 the chain (Fig. A1 in Appendix 1). WAIC scores showed a similar pattern favoring model 2 but

222 with some support for model 4, with values of 3766.0, 3596.0, 3769.3, and 3598.5 for models 1-

2234 , respectively.

224 Marginal posterior distributions of parameters for models 2 and 4 were similar (Table 1).

225 For model 4 , the sea-state coefficient $\beta_{8}$ was small in absolute value and the $95 \%$ credibility

226 interval included 0 , further indications that wind conditions in the range Beaufort 0-4 had little 
227 effect on the accuracy of group size estimation. The coefficient for $\log$ (photo count), $\beta_{1}$, was

$228<1.0$ (mean $0.80,95 \%$ credibility interval 0.76 to 0.83 for model 2 ), which meant that the

229 tendency to underestimate increased with group size. Species coefficients decreased in the order 230 mixed spotted-spinner, common, spotted, spinner, striped, and other (Fig. 3A). However, the

231 posterior distributions of species coefficients overlapped (Table 1), indicating that the differences

232 among species were modest. The random-effects coefficients were negatively correlated (mean $233 \rho=-0.79,95 \%$ credibility interval -0.59 to -0.91$)$.

234 Observers differed in accuracy of group size estimation (Fig. 3B). Among the 59

235 observers, some tended to underestimate and others tended to overestimate. For spotted dolphin

236 groups of $25,50,100$, and 500 animals, the observers with the lowest estimation tendency had

237 mean posterior estimates of $18,29,45$ and 132, respectively, while the observers with the highest

238 estimation tendency had mean posteriors of 42, 72, 125, and 585 (Table 2). The "average

239 observer" (actually four different observers, one for each of the four group sizes in Table 2) had

240 estimates of $25,44,78$, and 290 for spotted dolphin groups of 25, 50, 100, and 500, respectively.

241 Thus, over all observers, groups of 25 spotted dolphins were estimated accurately on average,

242 but the range among observers was from underestimation by $29 \%$ to overestimation by $66 \%$

243 (Table 2). There were similarly large ranges in accuracy among observers for larger groups: for

244 groups of $50,-43 \%$ to $+45 \%$; for groups of $100,-55 \%$ to $+25 \%$; and for groups of $500,-74 \%$ to

$245+17 \%$. The "average observer" underestimated spotted dolphin groups of 50, 100, and 500

246 animals by $11 \%, 22 \%$, and $42 \%$, respectively. We chose spotted dolphins for these numerical

247 comparisons because spotted dolphins were near the middle of the species effect (Table 1).

248 There would be less underestimation of group size for common dolphins and mixed groups of

249 spotted-spinner dolphins, and more underestimation of group size for spinner, striped, and other 
250 dolphins. Averaged over all species, the mean figures of underestimation were $<1 \%, 16 \%, 27 \%$, 251 and $47 \%$ for groups of $25,50,100$, and 500 animals.

252 The random-effects model allowed intercept and slope parameters to be estimated for 253 each observer (Fig. 4), constrained by the hierarchical assumptions of normal distributions and 254 correlation between slope and intercept. Visually, the greater importance of the observer effect 255 relative to the species effect can be judged by comparing Fig. 3B with Fig. 3A. Numerically, the 256 range of plausible values for observer intercepts $\left(1.5 \approx \pm 2 \sigma_{\alpha 0}\right)$ was greater than the range of 257 species effects $(\approx 0.4)$, based on the mean posterior values in Table 1 .

Accuracy decreased with group size (Fig. 5). Groups of 25 spotted dolphins were slightly 259 underestimated, but groups of 500 were severely underestimated. For groups of 25, 50, 100, and 260500 dolphins, posterior means for an out-of-sample observer (gray lines in Fig. 5) were 24.2, $26142.2,73.1$, and 264.4, respectively. To show conditional estimates, we used observer \#53 as an 262 example. The black lines in Fig. 5 for observer \#53 were slightly to the left of the gray lines 263 unconditioned on observer, indicating that this observer tended to underestimate more than the 264 average over all observers.

265 The posterior distributions of observer estimates were approximately normal on a natural 266 logarithmic scale (Fig. 5). The distributions were quite wide, illustrating the high uncertainty (or 267 low precision) in observer estimates of group size. Conditional estimates had higher precision 268 than unconditional estimates. Estimates made by a particular observer (observer \#53) for a 269 particular species (spotted dolphins) had slightly higher precision (less uncertainty) than 270 estimates by the same observer for an unknown species (compare thin dashed with thick solid 271 black lines in Fig. 5). Unconditional estimates for any observer or species had the least precision 
272 (thick gray lines in Fig. 5). The differences between conditional and unconditional estimates

273 were small, however, in the context of the overall high variability of group size estimates.

Predictions of dolphin group size from observer estimates

Conversely, given an observer estimate, predicted true group size was usually larger than

276 the estimate, especially for larger groups (Fig. 6). For observer estimates of 25, 50, 100, and 500

277 dolphins, posterior means were 26.0, 63.5, 154.0, and 1,194.5, respectively, for an out-of-sample

278 observer (gray lines in Fig. 6). As with posterior distributions of observer estimates given group

279 size, predicted group sizes conditional on observer and species had higher precision than

280 unconditional estimates (compare black and gray lines in Fig. 6). Because observer \#53 tended

281 to underestimate more than average, predicted group size was larger for this observer than for the 282 average over all observers.

283 Dolphin group size predicted from an observer estimate had high uncertainty.

284 Coefficients of variation for predicted group size conditional on species ranged from

285 approximately 0.7 to 0.9 (Table 3 ). Coefficients of variation for unconditional predictions were

286 even larger, ranging from 0.9 to 1.9 , due to the additional uncertainty of predicting group size for

287 an unknown species. Given an observer estimate of 100 dolphins, the $95 \%$ credibility interval

288 for the true size of the group ranged from 43 to 621 for a group of spotted dolphins, and from 37

289 to 776 for a group unconditional on species. Posterior distributions accurately captured the

290 uncertainty in predicting dolphin group size from an observer estimate (Appendix 5, Fig. A4).

291 The degree to which an observer estimate was increased to estimate true group size

292 depended on species. For an observer estimate of 25 dolphins, for example, the median

293 predicted group size was smaller than 25 for mixed spotted-spinner and common groups, and 
294 larger than 25 for spotted, spinner, striped, and other groups (Table 3). Because the

295 exponentiated posterior distributions were lognormal, means were larger than medians.

296 Therefore, we used the median (50\% quantile) as the best measure of central tendency for these

297 distributions, because there was equal probability of a value being higher or lower than the

298 median. Integrated over species and observer effects, estimates of 25, 50, 100, and 500 were

299 increased by $4 \%, 24 \%, 47 \%$, and $122 \%$, respectively, to obtain the medians of the posterior

300 distributions of predicted group size (Table 3). In other words, given an observer estimate of 500

301 dolphins, the most probable true size of the group would be more than twice that number.

\section{Discussion}

\section{Accuracy and precision}

The discrepancy between an observer estimate of dolphin group size and the true number

306 can be discussed in terms of two components: accuracy and precision. Accuracy is measured by

307 the difference between the true number and the mean of repeated observations. Inaccurate

308 measurement of group size leads to biased results. Precision is assessed by the random error

309 among repeated observations. Random error will be positive for some observations and negative

310 for others, but with a mean of zero. Low precision means high variance and greater uncertainty

311 in results.

312 We found that accuracy of dolphin group size estimates depended on group size,

313 observer, and species. Within the Beaufort 0-4 range of the calibration schools, Beaufort sea

314 state had less effect on accuracy, once group size and observer effects had been accounted for. 
There was a general tendency to underestimate dolphin group size, and this tendency

316 increased with group size. The coefficient of the log of photo count $\left(\beta_{1}\right.$, Table 1$)$ was $<1.0$,

317 which meant that large groups were underestimated more than small groups. Observer estimates

318 were accurate (on average) for dolphin groups of 25 animals, but were too low by $16 \%$ for

319 groups of 50 , too low by $27 \%$ for groups of 100 , and too low by $47 \%$ for groups of 500 (Fig. 5).

320 These estimates of accuracy averaged over all observers do not measure the accuracy of a

321 particular observer, nor the discrepancy between an observer estimate and true group size for a

322 particular group. Accuracy of dolphin group size estimation in this study applies within the

323 range of calibration school sizes with a reasonable number of samples, roughly between 10 and

3241000 animals (Fig. 3).

These results were broadly consistent with previous studies which showed that humans

326 tend to underestimate group sizes in wildlife studies (Caughley 1974, Bayliss and Yeomans

327 1990, Cogan and Diefenbach 1998). The rate of decline in accuracy with group size $\left(\beta_{1}=0.80\right.$,

328 Table 1) falls in the range of perceptual experiments measuring underestimation of the number of

329 dots on paper (Krueger 1972). Underestimation of large groups may have a physiological basis

330 related to eye movement; estimation of small groups (about 10 or fewer objects) does not have

331 this negative bias and seems to involve a different perceptual mechanism (Binda et al. 2011).

The degree of underestimation also varied by species. For the six species categories in

333 this study, dolphin group size estimates were lower in the order: mixed spotted-spinner,

334 common, spotted, spinner, striped, and other (Table 1, Fig. 3A). This order of species

335 coefficients corresponded roughly to mean group size among the six species groups, with mixed

336 spotted-spinner and common dolphin groups being largest, and striped and other dolphin groups 
337 smallest. This correspondence suggests that the effects of group size and species were somewhat 338 confounded.

Accuracy varied among the 59 observers. While there was an overall tendency to

340 underestimate dolphin group size, some observers had a stronger tendency to underestimate,

341 while others had a tendency to overestimate (Table 2). The random-effects model allowed the

342 estimation of separate effects for each observer (Fig. 3B), but connected the observers as a group

343 and allowed the tendency of all observers together to support estimation for each single observer

344 (Fig. 4). A random-effects model is often understood in terms of "partial pooling." It represents

345 an intermediate approach between complete pooling (treating all observers as a single group, Fig.

346 1A) and no pooling (treating each observer independently, Fig. 1B). The random-effects

347 approach spans a range of models between these extremes, and includes complete pooling and

348 complete separation as special cases at the limits (Gelman and Hill 2007). The degree of pooling

349 is related to the amount of shrinkage of individual effects toward the mean (Gelman and Pardoe 350 2006).

Precision of observer estimates of dolphin group size was strikingly low (Fig. 5). For a

352 group of 100 dolphins, for example, estimates could range from about 30 to 200 with $95 \%$

353 probability. Regardless of an observer's accuracy, it was common for the observer to estimate

$35450 \%$ high for one group and 50\% low for the next. As a consequence, there was high variability

355 among the independent observer estimates of group size, both for calibration schools as well as

356 for non-calibration dolphin groups. The mean CV among observer estimates was 0.4 across a

357 wide range of group sizes. Clearly, estimating the size of a dolphin group is a challenging task.

\section{$358 \quad$ Statistical issues}


As a measure of true group size, we used the mean of photo counts by three independent

360 readers. A binomial moment estimator has been proposed for repeated counts with imperfect

361 detection, i.e., false negatives (DasGupta and Rubin 2005, Walsh et al. 2009), but in our study

362 variation among counts of the three readers was also due to false positives. Large tuna, which

363 frequently accompany dolphin groups in the eastern tropical Pacific, can be mistaken for a

364 submerged dolphin in the photographs. Splashes and reflections might also be counted as a

365 partially hidden dolphin.

RJMCMC and WAIC are two fully Bayesian approaches to model selection (Hooten and

367 Hobbs 2015). RJMCMC treats the model itself as an additional unknown parameter to be

368 estimated, while WAIC is a score function based on the predictive ability of the model. Both

369 indicated that the accuracy of dolphin group size estimates varied by observer and species

370 (model 2). There was little posterior support for model 4, which included Beaufort sea state

371 (Fig. 2). The posterior odds of models 2 and 4 (the Bayes factor, Kass and Raftery 1995) was

37260.6 , indicating strong support for model 2 over model 4. The WAIC difference of 2.5 also

373 indicated support of model 2 over model 4. If sea state was modeled as a categorical variable,

374 model 4 had a posterior probability of zero (it was never selected in the RJMCMC algorithm),

375 but if sea state was modeled as a continuous variable, model 4 was selected $2 \%$ of the time (Fig.

376 2, Fig. A1 in Appendix 1). Thus, it appeared that modeling sea state as a continuous variable

377 rather than as separate factor variables was a more parsimonious approach. As there was little

378 support for model 4, and because parameter estimates were similar for models 2 and 4 (Table 1),

379 we focused on model 2 for inference and did not use model-averaged estimates.

381 inference regardless of observer and/or species, by integrating over observer and species effects. 
382 The estimation tendency of a new, out-of-sample observer included the uncertainty of not

383 knowing which observer, out of the "universe" of possible observers with different estimation

384 tendencies, might be chosen. Such estimates unconditional for observer and species are shown

385 as gray lines in Figs. 5 and 6. The greater uncertainty of the unconditional estimates is indicated

386 by the wider probability distributions in those figures, relative to the conditional estimates shown

387 with black lines.

388 Application of results

389 To obtain the best estimates of group size, we can use the estimation tendencies revealed

390 in this study to adjust observer estimates of dolphin group size. We wish to predict true group

391 size, given an observer estimate. The Bayesian approach allowed us to solve this inverse

392 problem with proper accounting of uncertainty. Since, for groups larger than about 25 dolphins,

393 there was a tendency to underestimate group size, predictions of true group size tended to be

394 larger than the estimate (Fig. 6). Because the degree of underestimation depended on group size,

395 species, and observer, the amount that a group size estimate had to be increased to predict true

396 group size also depended on group size, species, and observer (Table 3). The amount that an

397 estimate had to be increased could be substantial. For example, a group size estimate of 100

398 dolphins had to be increased by $47 \%$ to obtain the unconditional best (median) estimate of true

399 group size.

400 Because an estimate of group size had low precision, predicted group size based on an

401 estimate also had low precision. Posterior distributions had CVs of approximately 0.7 to 0.9 for

402 groups of known species, and 0.9 to 1.9 for groups of any species (Table 3). For an out-of-

403 sample observer estimate of 25 dolphins, for example, median predicted true group size was 25.9 
404 animals (accuracy was good), but the $50 \%$ credibility interval extended from 16 to 42 dolphins, 405 and the $95 \%$ credibility interval from 6 to 111 dolphins (Table 3 ). This source of uncertainty is 406 usually ignored in distance sampling analyses, although Gerrodette and Forcada (2005) included 407 uncertainty in group size through a bootstrap procedure. Most line-transect analyses compute the 408 variance in expected group size from the sizes of the observed groups.

On cetacean line-transect surveys conducted by the Southwest Fisheries Science Center,

410 three independent estimates of group size are recorded for each sighting. For the best estimate of 411 group size, Gerrodette and Forcada (2005) used an average of the three calibration-adjusted 412 observer estimates, weighted by the inverse of the group size estimation variance of each 413 observer. The value of making several independent estimates of group size will be examined in 414 a future paper.

Given our findings of inaccuracy for groups larger than 25 dolphins and low precision for 416 groups of all sizes, it is worth noting that the estimates of group size in this study were a selected 417 set of estimates made in optimal circumstances. Each group was approached with the specific 418 objective of obtaining group size estimates, the observers had good views of the entire group, 419 and the ship remained with the group until the observers had made their best possible estimates. 420 Almost certainly the behavior of dolphin groups affects the accuracy and precision of group size 421 estimates, but our set of calibration schools consisted of well-behaved groups that could be 422 observed and photographed in their entirety. Accuracy and precision may be lower for groups estimated in less optimal conditions.

424 Schwarz et al. (2010) found that estimates of delphinid group sizes were 58\% lower when the 425 ship did not approach groups (passing mode) than when it did (closing mode). Barlow et al. 
426 (1997) also found that group size estimates were smaller in passing mode. Barlow and Taylor

427 (2005) found that an extended 90-min period of observation improved group size estimates of 428 asynchronously diving sperm whales (Physeter microcephalus). The position of the observer

429 may also matter. The estimates of group size in this study were made from a platform

430 approximately $10 \mathrm{~m}$ above the water. The estimation tendencies reported here may not apply to

431 other situations, such as estimates made from higher or lower platforms on a ship, or estimates

432 made from land at various elevations and distances to sightings. Caughley et al. (1976) found

433 that the accuracy of aerial counts varied with aircraft speed, height, and observer.

We conclude with two recommendations for studies that depend on estimates of cetacean

435 group size. First, we recommend training to improve group size estimation. Although we were

436 not able to measure how our pre-cruise training affected observers' estimates, we believe that the

437 training had a positive effect. Training may include displays of groups of objects of known size,

438 and instructions on estimating group size by counting subgroups of multiple animals. Second,

439 we recommend assessment of accuracy and precision of group size estimation under the

440 particular conditions of a study. The large budget of this study is unlikely to be replicated, but

441 digital photography by drones is a more economical and much safer option today. Laake et al.

442 (2012) used two observer teams to assess the accuracy of pod size estimates for migrating gray

443 whales (Eschrichtius robustus). Although pod size was usually only one or two animals,

444 correcting pod size estimates had an important effect on abundance estimates and inferred

445 population trajectory.

446 If a study is unable to assess accuracy of group size estimates, the results of this study can

447 be applied with appropriate caution. We have noted that biases might be different for group size

448 estimates made under other conditions, such as greater distances. One of our central results was 
449 that people varied widely in their group size estimation tendencies; therefore, the ideal is to 450 calibrate particular individual observers. However, the random-effects model for the observer 451 effect allowed inference for observers outside this study. Table 3 and Figures 5 and 6 show 452 posterior distributions for a new, out-of-sample observer - that is, accuracy and precision of 453 group size estimates which include the uncertainty of not knowing which observer, out of the 454 large number of possible observers with different estimation tendencies, might have been chosen. 455 Unless more specific information can be obtained, it would be reasonable to assume that the 456 estimation tendencies of the 59 observers in this study are representative of all observers. 
458 We thank all the marine mammal observers who made the group size estimates, the aerial 459 photographers and NOAA helicopter pilots, the captains and crews of the NOAA research 460 vessels, the support staff at the Southwest Fisheries Science Center, and the Government of 461 Mexico for flight permission in 2006. The photo counts were made by Jim Gilpatrick, Robin 462 Westlake, Morgan Lynn, and Katie Cramer of the SWFSC. The manuscript benefitted from 463 comments by Jim Gilpatrick, Jeffrey Moore, and two anonymous reviewers. 
Barlow, J. 1997. Preliminary estimates of cetacean abundance off California, Oregon and Washington based on a 1996 ship survey and comparisons of passing and closing modes. Southwest Fisheries Science Center Administrative Report LJ-97-11. 25 p.

Barlow, J. and K. A. Forney. 2007. Abundance and population density of cetaceans in the California Current ecosystem. Fishery Bulletin 105: 509-526.

Barlow, J. and B. L. Taylor. 2005. Estimates of sperm whale abundance in the northeastern temperate Pacific from a combined acoustic and visual survey. Marine Mammal Science 21: 429-445.

Bayliss, P. and K. M. Yeomans. 1990. Use of low-level aerial photography to correct bias in aerial survey estimates of magpie goose, and whistling duck density in the Northern Territory. Australian Wildlife Research 17: 1-10.

Binda, P., M. Concetta Morrone, J. Ross and D. C. Burr. 2011. Underestimation of perceived number at the time of saccades. Vision Research 51: 34-42.

Borchers, D. L. and K. P. Burnham. 2004. General formulation for distance sampling. Pages 6-

483 Caughley, G., R. Sinclair and D. Scott-Kemmis. 1976. Experiments in aerial survey. Journal of 484 Wildlife Management 40: 290-300. 
Cogan, R. D. and D. R. Diefenbach. 1998. Effect of undercounting and model selection on a sightability-adjustment estimator for elk. The Journal of Wildlife Management 62: 269279.

DasGupta, A. and H. Rubin. 2005. Estimation of binomial parameters when both $n, p$ are unknown. Journal of Statisical Planning and Inference 130: 391-404.

Erwin, R. M. 1982. Observer variability in estimating numbers: an experiment. Journal of Field Ornithology 53: 159-167.

Gelman, A. and J. Hill 2007. Data analysis using regression and multilevel/hierarchical models. Cambridge University Press, Cambridge, MA.

Gelman, A., J. Hwang and A. Vehtari. 2014. Understanding predictive information criteria for Bayesian models. Statistics and Computing 24: 997-1016.

Gelman, A. and I. Pardoe. 2006. Bayesian measures of explained variance and pooling in multilevel (hierarchical) models. Technometrics 48: 241-251.

Gelman, A., G. O. Roberts and W. R. Gilks. 1996. Efficient Metropolis jumping rules. Bayesian Statistics 5: 599-607.

Gerrodette, T. and J. Forcada. 2005. Non-recovery of two spotted and spinner dolphin populations in the eastern tropical Pacific Ocean. Marine Ecology Progress Series 291: 121.

Gilpatrick, J. W., Jr. 1993. Method and precision in estimation of dolphin school size with vertical aerial photography. Fishery Bulletin 91: 641-648.

Green, P. J. 1995. Reversible jump Markov Chain Monte Carlo computation and Bayesian model determination. Biometrika 82: 711-732. 
507 Hastings, W. K. 1970. Monte Carlo sampling methods using Markov chains and their

$508 \quad$ applications. Biometrika 57: 97-109.

509 Hooten, M. B. and N. T. Hobbs. 2015. A guide to Bayesian model selection for ecologists.

$510 \quad$ Ecological Monographs 85: 3-28.

511 Kass, R. E. and A. E. Raftery. 1995. Bayes factors. Journal of the American Statistical

$512 \quad$ Association 90: 773-795.

513 King, R., B. J. T. Morgan, O. Gimenez and S. P. Brooks 2009. Bayesian analysis for population 514 ecology. Chapman \& Hall/CRC.

515 Krueger, L. E. 1972. Perceived numerosity. Perception \& Psychophysics 11: 5-9.

516 Laake, J. L., A. E. Punt, R. Hobbs, M. Ferguson, D. Rugh and J. Breiwick. 2012. Gray whale 517 southbound migration surveys 1967-2006: An integrated re-analysis. Journal of Cetacean $518 \quad$ Research and Management 12: 287-306.

519 Lunn, D., C. Jackson, N. Best, A. Thomas and D. Spiegelhalter 2013. The BUGS book: A 520 practical introduction to Bayesian analysis. CRC Press, Boca Raton, FL.

521 Lunn, D. J., A. Thomas, N. Best and D. Spiegelhalter. 2000. WinBUGS -- a Bayesian modelling 522 framework: Concepts, structure, and extensibility. Statistics and Computing 10: 325-337.

523 Metropolis, N., A. Rosenbluth, M. N. Rosenbluth and A. Teller. 1953. Equation of state 524 calculations by fast computing machines. The Journal of Chemical Physics 21:1087$525 \quad 1092$.

526 Millar, R. B. 2009. Comparison of hierarchical Bayesian models for overdispersed count data 527 using DIC and Bayes' factors. Biometrics 65: 962-969. 
528 Oedekoven, C. S., S. T. Buckland, M. L. Mackenzie, R. King, K. O. Evans and L. W. Burger, Jr.

529 2014. Bayesian methods for hierarchical distance sampling models. Journal of

$530 \quad$ Agricultural, Biological, and Environmental Statistics 19: 219-239.

531 Plummer, M. 2008. Penalized loss functions for Bayesian model comparison. Biostatistics 9:

$532 \quad 523-539$.

533 Ryan, P. G. and J. Cooper. 1989. Observer precision and bird conspicuousness during counts of 534 birds at sea. South African Journal of Marine Science 8: 271-276.

535 Schwarz, L. K., T. Gerrodette and F. I. Archer. 2010. Comparison of closing and passing mode

536 from a line-transect survey of delphinids in the eastern Tropical Pacific Ocean. Journal of

537 Cetacean Research and Management 11:253-265.

538 Spiegelhalter, D. J., N. G. Best, B. P. Carlin and A. Van Der Linde. 2002. Bayesian measures of 539 model complexity and fit. Journal of the Royal Statistical Society, B 64: 583-639.

540 Vehtari, A., A. Gelman and J. Gabry. 2016. Practical Bayesian model evaluation using leave541 one-out cross-validation and WAIC. Statistics and Computing 26: 1-20.

542 Walsh, D. P., C. F. Page, H. Campa, Iii, S. R. Winterstein and D. E. Beyer, Jr. 2009.

543 Incorporating estimates of group size in sightability models for wildlife. The Journal of $544 \quad$ Wildlife Management 73: 136-143.

545 Watanabe, S. 2010. Asymptotic equivalence of Bayes cross validation and widely applicable 546 information criterion in singular learning theory. Journal of Machine Learning Research $547 \quad 11: 3571-3594$. 
550 Table 1. Marginal posterior distributions of parameters for the two models with posterior

551 support. Distributions are summarized by means, standard deviations (SD) and three quantiles.

552 All parameters had uniform prior distributions. $\mathrm{RE}=$ random effects for observers. See Eq. 1

553 for definitions of parameters.

\begin{tabular}{|l|c|c|c|c|c|c|c|c|c|c|}
\hline & \multicolumn{5}{|c|}{ Model 2} & \multicolumn{5}{c|}{ Model 4 } \\
\hline \multicolumn{1}{|c}{ Parameter } & Mean & SD & $2.5 \%$ & $50 \%$ & $97.5 \%$ & Mean & SD & $2.5 \%$ & $50 \%$ & $97.5 \%$ \\
\hline Log(photo count), $\beta_{1}$ & 0.796 & 0.018 & 0.761 & 0.796 & 0.832 & 0.797 & 0.018 & 0.760 & 0.798 & 0.831 \\
\hline Sp: spotted-spinner, $\beta_{2}$ & 0.805 & 0.087 & 0.635 & 0.803 & 0.974 & 0.818 & 0.087 & 0.653 & 0.816 & 0.995 \\
\hline Sp: common, $\beta_{3}$ & 0.757 & 0.084 & 0.592 & 0.755 & 0.926 & 0.776 & 0.087 & 0.613 & 0.774 & 0.951 \\
\hline Sp: spotted, $\beta_{4}$ & 0.656 & 0.082 & 0.497 & 0.654 & 0.816 & 0.674 & 0.083 & 0.521 & 0.672 & 0.843 \\
\hline Sp: spinner, $\beta_{5}$ & 0.603 & 0.088 & 0.433 & 0.604 & 0.778 & 0.620 & 0.091 & 0.448 & 0.618 & 0.806 \\
\hline Sp: striped, $\beta_{6}$ & 0.513 & 0.075 & 0.370 & 0.511 & 0.660 & 0.531 & 0.078 & 0.386 & 0.530 & 0.687 \\
\hline Sp: other, $\beta_{7}$ & 0.423 & 0.076 & 0.273 & 0.424 & 0.576 & 0.442 & 0.079 & 0.291 & 0.440 & 0.601 \\
\hline Beaufort sea state, $\beta_{8}$ & na & na & na & na & na & -0.010 & 0.010 & -0.030 & -0.010 & 0.010 \\
\hline SD intercept RE, $\sigma_{\alpha 0}$ & 0.382 & 0.073 & 0.245 & 0.377 & 0.534 & 0.385 & 0.073 & 0.252 & 0.381 & 0.540 \\
\hline SD slope RE, $\sigma_{\alpha 1}$ & 0.104 & 0.017 & 0.073 & 0.102 & 0.138 & 0.104 & 0.017 & 0.073 & 0.103 & 0.139 \\
\hline Correlation RE, $\rho$ & -0.792 & 0.085 & -0.912 & -0.808 & -0.586 & -0.792 & 0.088 & -0.912 & -0.809 & -0.578 \\
\hline SD model, $\sigma_{\varepsilon}$ & 0.497 & 0.008 & 0.482 & 0.497 & 0.512 & 0.497 & 0.007 & 0.482 & 0.497 & 0.511 \\
\hline
\end{tabular}

554 
556 Table 2. Summary of estimation tendencies among observers. For each group size, the entries in 557 the table show the distribution of the means of the posteriors of the 59 observers for estimates of 558 a group of spotted dolphins. "Mean diff.", "Min diff." and "Max diff." are the differences

559 between the mean (or minimum or maximum) of the mean observer estimates and true group 560 size, expressed as percentages of group size.

\begin{tabular}{|c|c|c|c|c|c|c|c|c|c|}
\hline \multirow{2}{*}{$\begin{array}{l}\text { Group } \\
\text { size }\end{array}$} & \multicolumn{6}{|c|}{ Distribution of means of observer estimates } & \multirow{2}{*}{$\begin{array}{c}\text { Mean } \\
\text { diff. }\end{array}$} & \multirow{2}{*}{$\begin{array}{l}\text { Min } \\
\text { diff. }\end{array}$} & \multirow{2}{*}{$\begin{array}{l}\text { Max } \\
\text { diff. }\end{array}$} \\
\hline & Mean & Min & $25 \%$ & $50 \%$ & $75 \%$ & Max & & & \\
\hline 25 & 25.4 & 17.7 & 21.2 & 25.9 & 28.7 & 41.5 & $2 \%$ & $-29 \%$ & $66 \%$ \\
\hline 50 & 44.4 & 28.5 & 35.5 & 45.0 & 51.1 & 72.4 & $-11 \%$ & $-43 \%$ & $45 \%$ \\
\hline 100 & 77.7 & 44.9 & 60.3 & 78.3 & 89.1 & 124.6 & $-22 \%$ & $-55 \%$ & $25 \%$ \\
\hline 500 & 289.7 & 131.7 & 201.8 & 293.0 & 355 & 585.3 & $-42 \%$ & $-74 \%$ & $17 \%$ \\
\hline
\end{tabular}


562 Table 3. Predicted dolphin group sizes given observer estimates of 25, 50, 100, and 500 animals 563 by a new (out-of-sample) observer, for six dolphin species and integrated over species ("any 564 species"). Posterior distributions have been exponentiated to show values on the scale of the 565 number of dolphins. Distributions of predicted group size are approximately lognormal, and are 566 summarized by means, standard deviations (SD), coefficients of variation (CV) and five 567 quantiles. "Difference" is the difference between the median (the $50 \%$ quantile) of predicted 568 group size and observer estimate, expressed as a percentage of the observer estimate. 


\begin{tabular}{|c|c|c|c|c|c|c|c|c|c|c|}
\hline \multirow{2}{*}{$\begin{array}{l}\text { Observer } \\
\text { estimate }\end{array}$} & \multirow{2}{*}{$\begin{array}{c}\text { Dolphin } \\
\text { species }\end{array}$} & \multicolumn{8}{|c|}{ Predicted group size } & \multirow{2}{*}{$\begin{array}{l}\text { Differ- } \\
\text { ence }\end{array}$} \\
\hline & & Mean & SD & $\mathrm{CV}$ & $2.5 \%$ & $25 \%$ & $50 \%$ & $75 \%$ & $97.5 \%$ & \\
\hline \multirow{7}{*}{25} & spotted-spinner & 26.4 & 19.6 & 0.74 & 5.3 & 13.3 & 21.2 & 33.3 & 78.2 & $-15 \%$ \\
\hline & common & 28.2 & 21.1 & 0.75 & 5.5 & 14.3 & 22.6 & 35.8 & 83.4 & $-10 \%$ \\
\hline & spotted & 32.3 & 24.4 & 0.76 & 6.6 & 16.3 & 25.9 & 40.8 & 95.7 & $4 \%$ \\
\hline & spinner & 34.6 & 26.3 & 0.76 & 6.9 & 17.4 & 27.7 & 43.6 & 103.6 & $11 \%$ \\
\hline & striped & 39.0 & 29.1 & 0.75 & 7.9 & 19.9 & 31.4 & 49.3 & 113.9 & $26 \%$ \\
\hline & other & 43.8 & 33.3 & 0.76 & 8.8 & 22.1 & 35.2 & 55.3 & 130.3 & $41 \%$ \\
\hline & any species & 34.1 & 30.0 & 0.88 & 6.2 & 16.1 & 25.9 & 42.0 & 110.6 & $4 \%$ \\
\hline \multirow{7}{*}{50} & spotted-spinner & 66.6 & 50.4 & 0.76 & 13.9 & 33.8 & 53.5 & 83.6 & 196.6 & $7 \%$ \\
\hline & common & 70.7 & 53.2 & 0.75 & 14.7 & 36.1 & 56.7 & 88.7 & 209.9 & $13 \%$ \\
\hline & spotted & 81.1 & 61.6 & 0.76 & 17.0 & 41.2 & 65.1 & 101.4 & 239.7 & $30 \%$ \\
\hline & spinner & 87.3 & 66.7 & 0.76 & 18.1 & 44.3 & 70.3 & 110.1 & 256.5 & $41 \%$ \\
\hline & striped & 98.6 & 75.2 & 0.76 & 20.2 & 49.9 & 78.6 & 123.9 & 294.5 & $57 \%$ \\
\hline & other & 110.6 & 84.1 & 0.76 & 23.1 & 56.1 & 88.8 & 138.8 & 325.8 & $78 \%$ \\
\hline & any species & 84.5 & 79.6 & 0.94 & 15.7 & 38.8 & 62.1 & 102.6 & 286.9 & $24 \%$ \\
\hline \multirow{7}{*}{100} & spotted-spinner & 167.2 & 128.0 & 0.77 & 34.7 & 84.6 & 133.2 & 208.8 & 502.4 & $33 \%$ \\
\hline & common & 179.5 & 137.4 & 0.77 & 37.7 & 90.9 & 143.3 & 224.6 & 538.7 & $43 \%$ \\
\hline & spotted & 206.2 & 160.0 & 0.78 & 43.4 & 103.9 & 163.4 & 257.5 & 620.8 & $63 \%$ \\
\hline & spinner & 222.2 & 175.1 & 0.79 & 46.7 & 111.4 & 175.4 & 276.6 & 680.3 & $75 \%$ \\
\hline & striped & 247.3 & 191.0 & 0.77 & 52.7 & 124.6 & 195.8 & 308.9 & 748.2 & $96 \%$ \\
\hline & other & 281.7 & 219.6 & 0.78 & 59.4 & 142.1 & 223.3 & 349.7 & 847.5 & $123 \%$ \\
\hline & any species & 211.2 & 241.4 & 1.14 & 37.4 & 90.2 & 147.4 & 248.5 & 776.3 & $47 \%$ \\
\hline \multirow{7}{*}{500} & spotted-spinner & 1466.7 & 1215.5 & 0.83 & 305.0 & 723.5 & 1137.9 & 1810.4 & 4557.1 & $128 \%$ \\
\hline & common & 1581.4 & 1335.3 & 0.84 & 326.1 & 766.7 & 1218.6 & 1956.9 & 4981.8 & $144 \%$ \\
\hline & spotted & 1801.7 & 1518.8 & 0.84 & 367.9 & 876.1 & 1386.9 & 2227.2 & 5629.0 & $177 \%$ \\
\hline & spinner & 1941.8 & 1651.7 & 0.85 & 400.7 & 938.3 & 1490.8 & 2399.8 & 6192.8 & $198 \%$ \\
\hline & striped & 2181.9 & 1972.4 & 0.90 & 447.1 & 1057.2 & 1674.9 & 2689.5 & 6898.4 & $235 \%$ \\
\hline & other & 2483.6 & 2145.0 & 0.86 & 498.4 & 1190.7 & 1892.8 & 3053.4 & 7876.5 & $279 \%$ \\
\hline & any species & 1944.2 & 3600.3 & 1.85 & 250.6 & 626.9 & 1108.2 & 2103.6 & 8468.8 & $122 \%$ \\
\hline
\end{tabular}


575 Fig. 1: Dolphin group size calibration data plotted on logarithmic scales. (A) Photo counts and 576 observer estimates of group size for 434 calibration schools. The size of each group was

577 estimated independently by multiple (usually 6) shipboard observers. The dashed line is a 578 regression of $\log ($ observer estimate $)$ on $\log ($ photo count $)$, while the solid gray line is a 1:1

579 relationship. (B) Regressions of $\log$ (observer estimate) on $\log$ (photo count) for each of the 59 580 observers.

581 Fig. 2. Prior and posterior probabilities of four models of dolphin group size estimation based on 582 RJMCMC. Differences among observers were modeled as random effects (RE) in all four 583 models; species and sea state were fixed effects.

584 Fig. 3. Estimates of (A) species and (B) observer effects on dolphin group size estimation. 585 Regression lines are based on means of posterior distributions.

586 Fig. 4. Posterior distributions of random effects for each observer for (A) intercept $\alpha_{0}$ and (B) 587 slope $\alpha_{1}$ (see Eq. 1). Points are means and lines are central 95\% credibility intervals.

588 Fig. 5. Posterior distributions of observer estimates for dolphin groups of 25, 50, 100, and 500 589 animals. Thin dashed lines are the distributions of estimates for a given observer (\#53) whose 590 tendencies were estimated in this study, for a given species (spotted dolphins). Thick black lines 591 are the distributions for the same observer for any species (integrated over species). Thick gray 592 lines are the distributions for a new, out-of-sample observer with unknown tendencies, for any 593 species (integrated over observers and species). The probability densities (vertical scale) of all 594 distributions are scaled relative to the maximum value. 
595 Fig. 6. Predicted dolphin group sizes given observer estimates of 25, 50, 100, and 500 animals.

596 Thin dashed lines are the distributions of group size for a given observer (\#53) whose tendencies

597 were estimated in this study, for a given species (spotted dolphins). Thick black lines are the

598 distributions for the same observer for any species (integrated over species). Thick gray lines are

599 the distributions for a new, out-of-sample observer with unknown tendencies, for any species

600 (integrated over observers and species). The probability densities (vertical scale) of all

601 distributions are scaled relative to the maximum density value.

602 
603
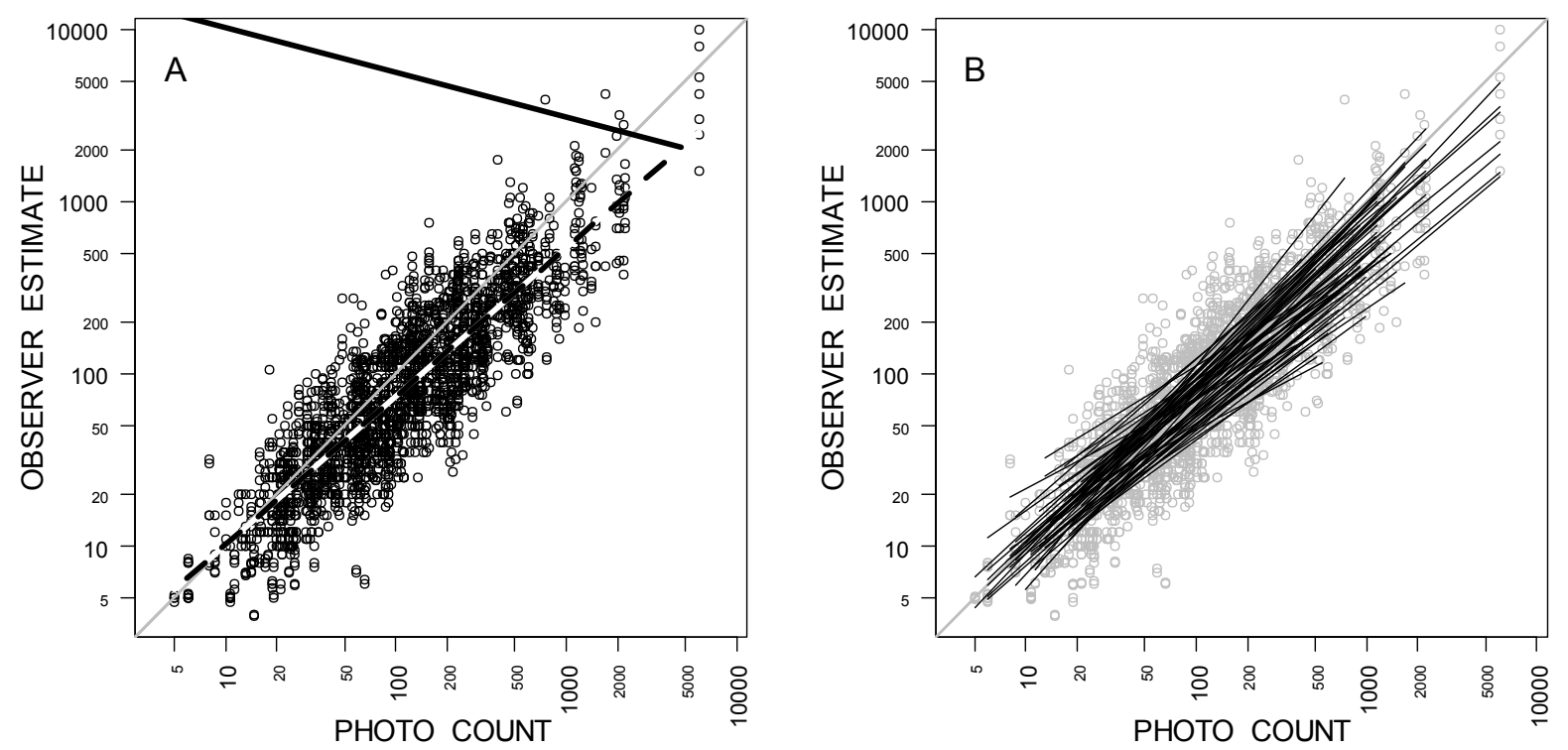

604 Fig. 1

605

606

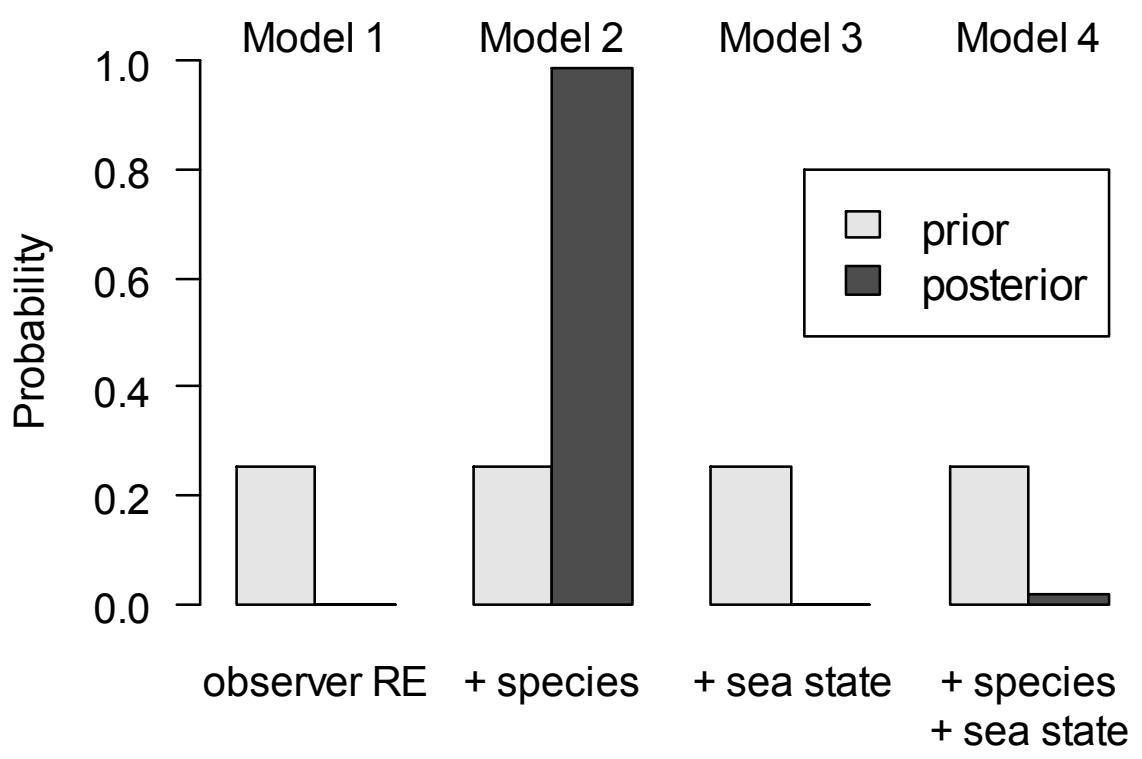

607

608 Fig. 2 
609
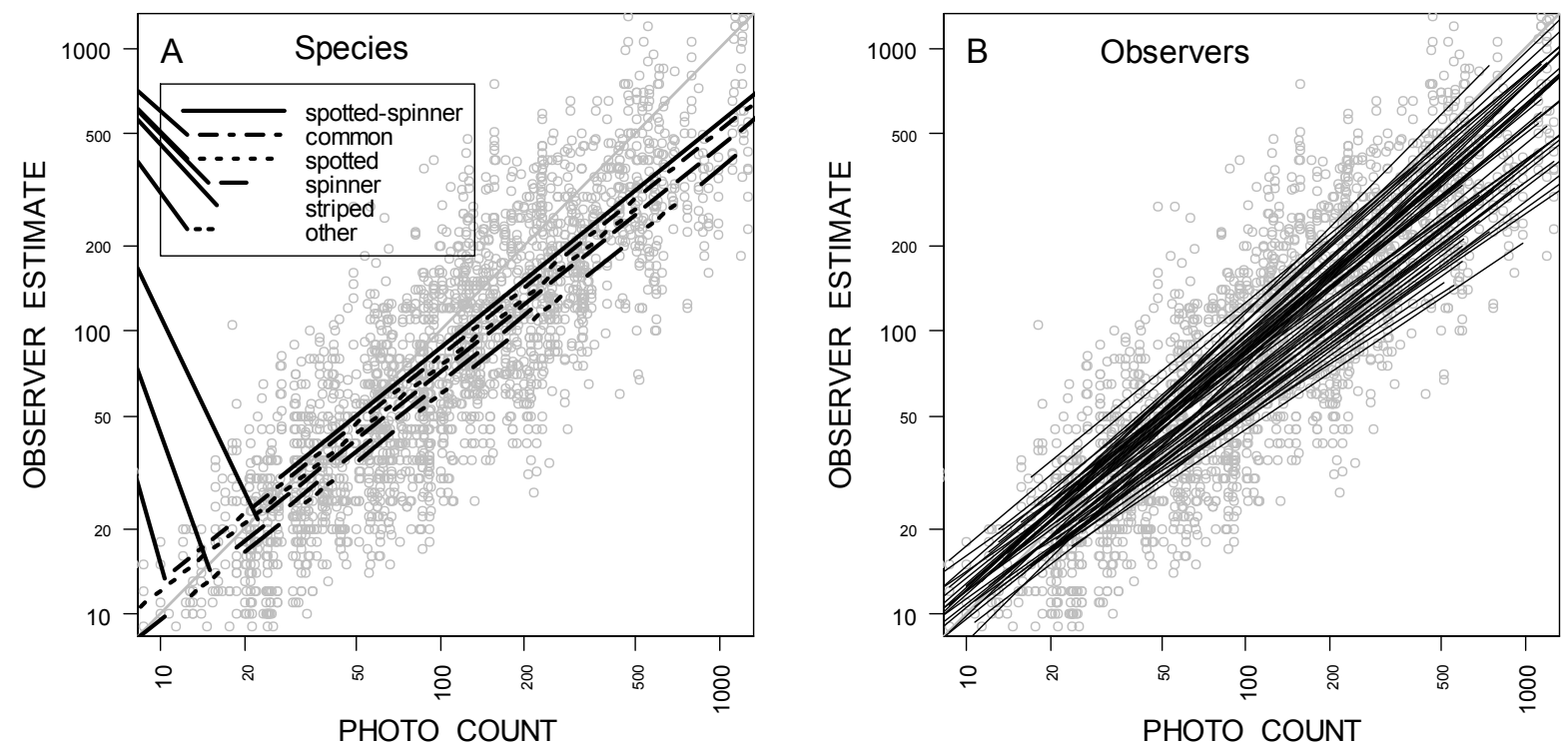

610 Fig. 3

611

612

613
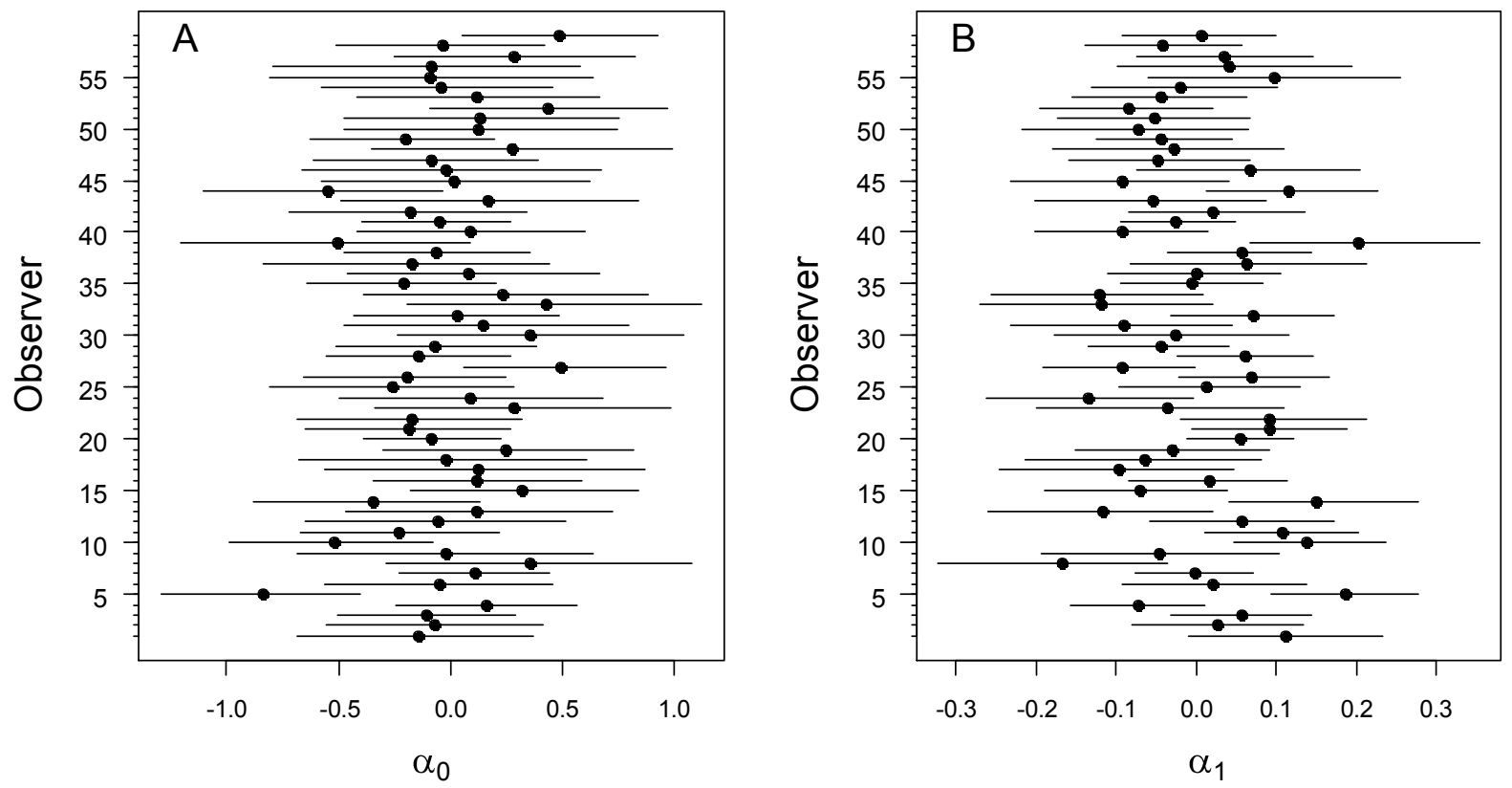

614

615 Fig. 4 


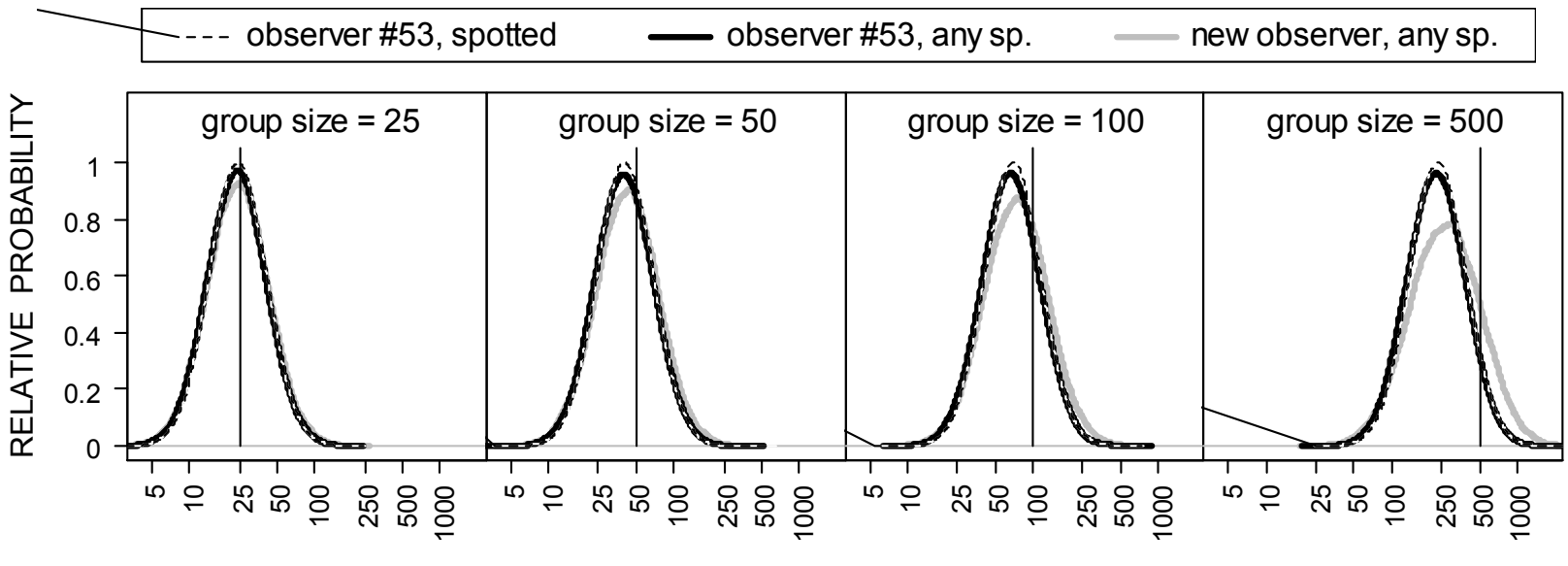

619 Fig. 5

620

621

622

623

624
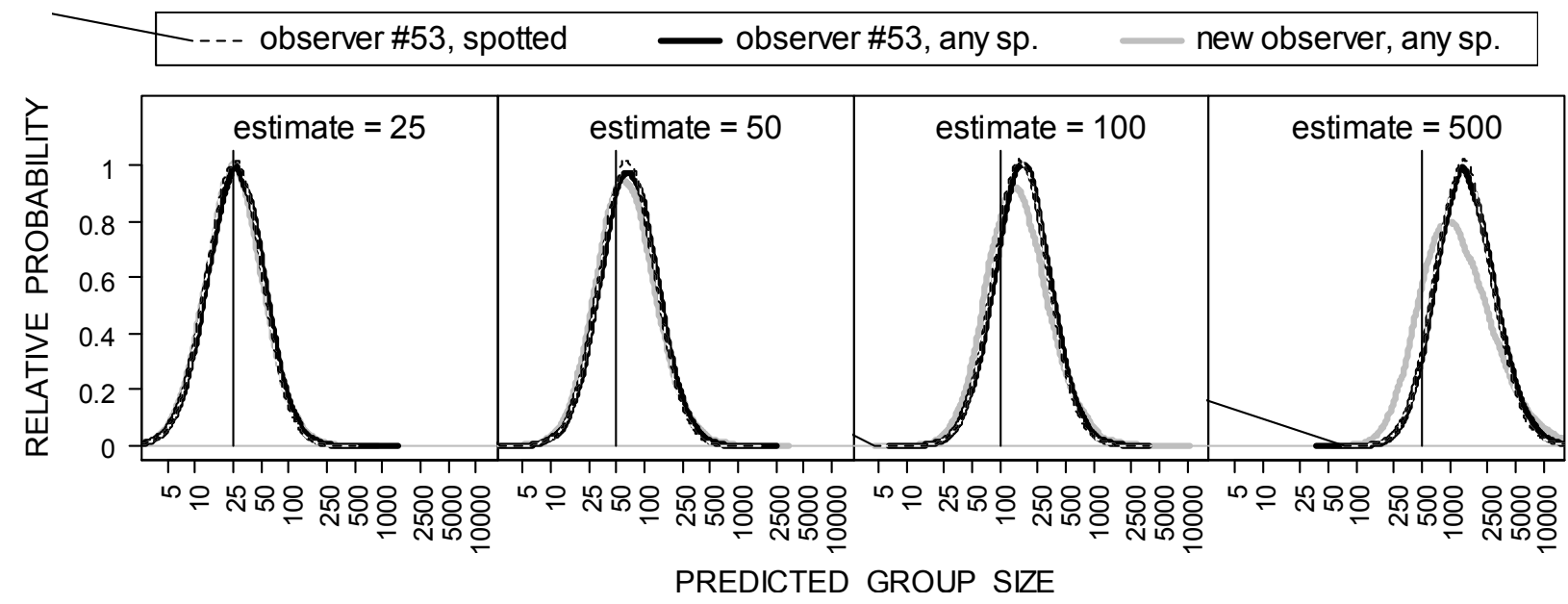

625 Fig. 6

626 
Random-effects models such as Eq. (1) can be implemented in a Bayesian framework using hierarchical models where each parameter, including the random-effects standard

630 deviations, are assumed to have a distribution. Markov Chain Monte Carlo (MCMC) simulation

631 can be used to obtain summary statistics of the posterior distributions of the parameters given the

632 data. To include model selection in our analysis, we treated the model itself as a parameter and

633 formed the joint posterior distribution of both parameters and models. An RJMCMC algorithm

634 (Green 1995) explored this posterior distribution. The RJMCMC algorithm represented a

635 random walk, where each iteration consisted of two steps: (1) the reversible jump (RJ) step

636 where we proposed to move to a different model (the between-model move), and (2) the

637 Metropolis-Hastings (MH) step where we updated the parameters from the current model (the

638 within-model move). We placed uniform priors on all parameters with an upper bound of 1 , and

639 a lower bound of -1 for coefficients and a lower bound of 0 for standard deviations.

All models included in the analysis contained the intercept and the log of the photo

641 counts as well as their corresponding random-effects coefficients (Eq. 1). Hence, the RJ step at

642 each iteration consisted of proposing to add or delete each of the two remaining covariates (sea-

643 state and species) in turn, depending on whether the covariate was in the current model or not.

644 Four different models were possible that differed only in the inclusion or exclusion of species

$645\left(\beta_{k}\right.$, with $\left.k=2, \ldots, 7\right)$ and sea state $\left(\beta_{8}\right)$ coefficients in Eq.(1): for model $1, \beta_{k}=0$ and $\beta_{8}=0$; for

$646 \operatorname{model} 2, \beta_{8}=0$; for model $3, \beta_{k}=0$; and for model 4 , both species and sea-state coefficients were

647 non-zero (full model) . A proposal to add a covariate to the model involved drawing random

648 samples from the respective proposal distributions for the parameters and accepting this proposal

649 based on the calculated acceptance probability (see, e.g., King et al. 2009 on how to obtain the 
650 acceptance probability). A proposal to delete a covariate from a model involved setting its

651 coefficients to zero and accepting this proposal based on the calculated acceptance probability.

652 The four models were considered equally likely a priori.

653 The MH step at each iteration consisted of updating the parameters that were currently in

654 the model using an MH update (Metropolis et al. 1953, Hastings 1970). This included the

655 coefficient associated with the log of the photo counts, the standard deviations associated with

656 the random effects and model errors as well as the coefficients for species and sea-state if these

657 covariates were in the current model. Furthermore, all random-effects coefficients were updated

658 during each iteration. In particular, this update involved a random walk single-update with

659 normal proposal distributions, where the mean was equal to the current value of the parameter

660 (or random-effects coefficient) and the standard deviations were fine-tuned during pilot tuning to

661 achieve appropriate acceptance rates (Gelman et al. 1996).

662 The chain was started with the full model and completed 210,000 iterations. We

663 discarded the first 10,000 as burn-in and thinned the chain by retaining every $50^{\text {th }}$ value, thus

664 obtaining a posterior sample of 4000 values. Posterior model probabilities were the fraction of

665 iterations that the chain spent in the respective model. Models 1 and 3 were never selected;

666 model 4 was selected $1.6 \%$ of the time consistently through the history of the chain (Fig. A1).

667 Similar results were obtained regardless of which model was used to initiate the chain. 


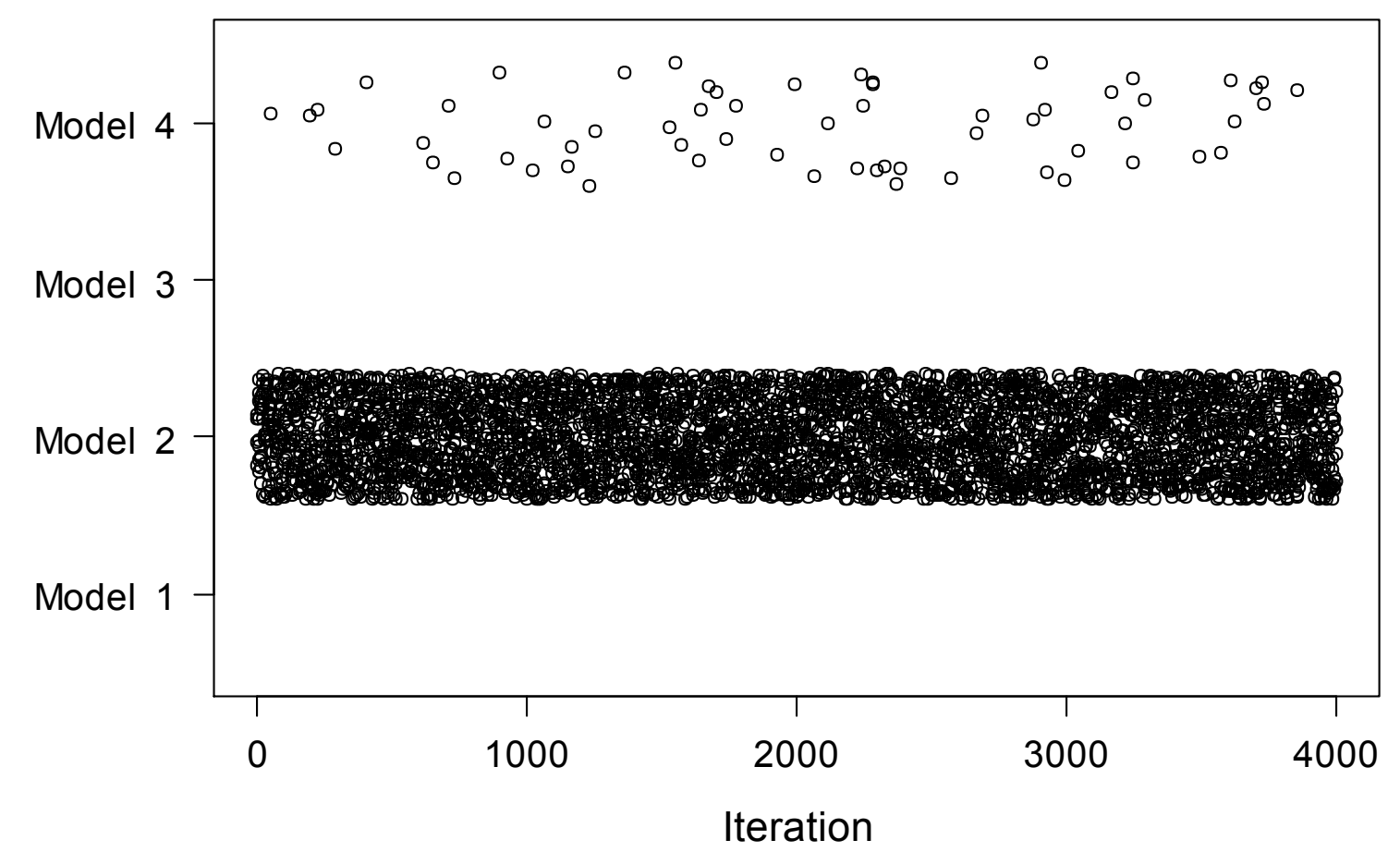

669

670 Fig. A1. Sequence of RJMCMC jumps among models after burn-in. Results were similar

671 regardless of which model was chosen to initiate the chain. To show separate points, random 672 values have been added to each point (jittering).

673 
674 Appendix 2. BUGS models and diagnostics for MCMC sampling

675 Each of the four variants of Eq. 1 was implemented in the BUGS language. Uniform

676 priors were specified for all parameters except the random-effects coefficients, which were

677 latent. Due to the large amount of data, specification of other priors, such as normal distributions

678 (lognormal distributions for variance parameters) with means far from values supported by the

679 data, had no effect on posterior distributions. For each model, we ran three chains of 120,000

680 iterations each, discarding the first 20,000 as burn-in from different random initial starting

681 values. For the remaining 100,000 iterations, we retained every $100^{\text {th }}$ value (thinning) to reduce

682 autocorrelation. Thus the final sample consisted of 1000 values for each of three chains. The

683 effective sample size for each parameter, calculated with R package coda, was near 1000,

684 indicating that autocorrelation was low. The chains were well-mixed for all parameters (Fig.

685 A2), and converged to similar values (Fig. A3). 


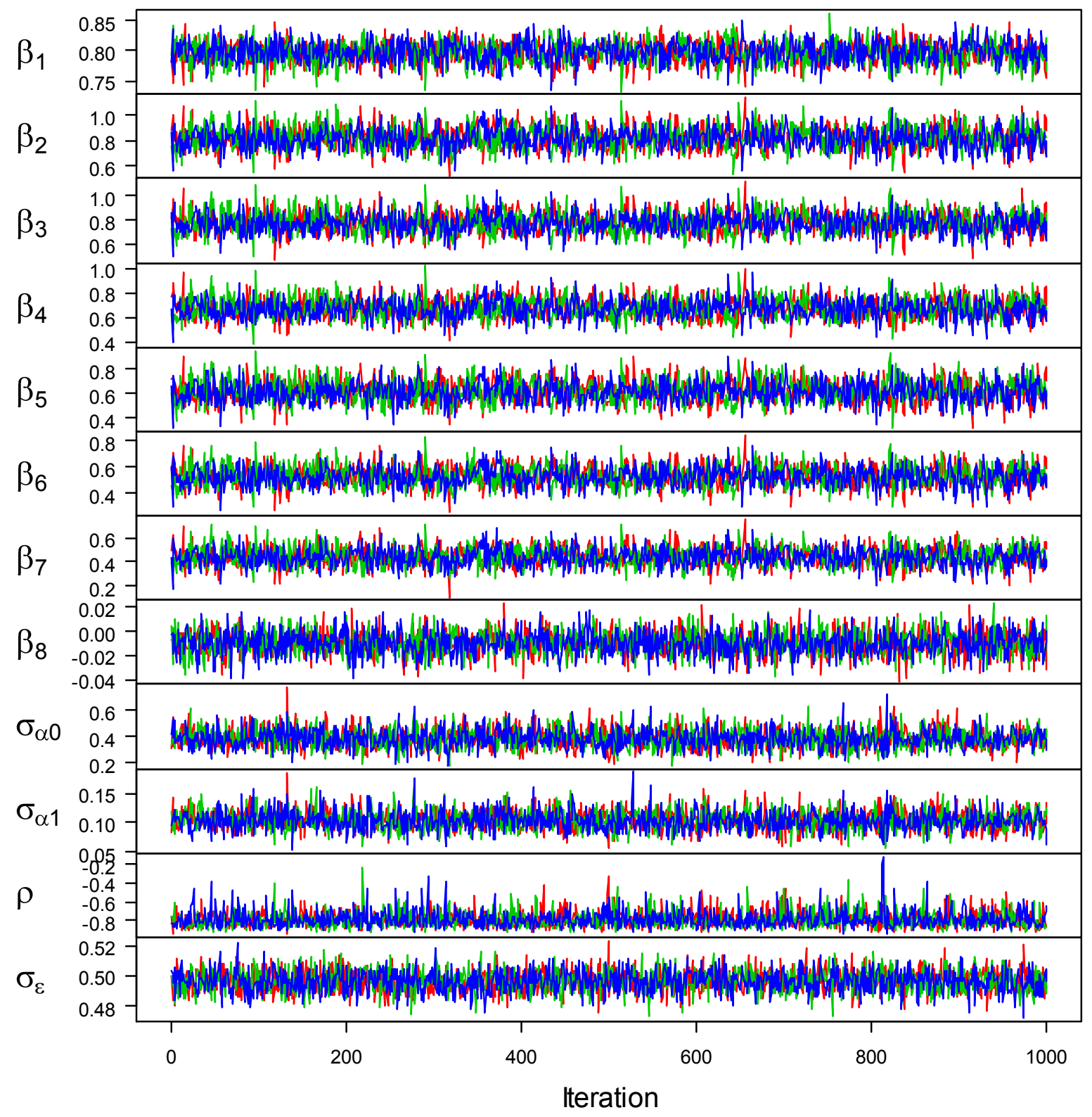

690 Fig. A2. Traces of posterior samples. Green, red and blue lines show three independent MCMC

691 chains of 1000 iterations each, with different initial values. See Eq. 1 and Table 1 for definitions

692 of parameters. 

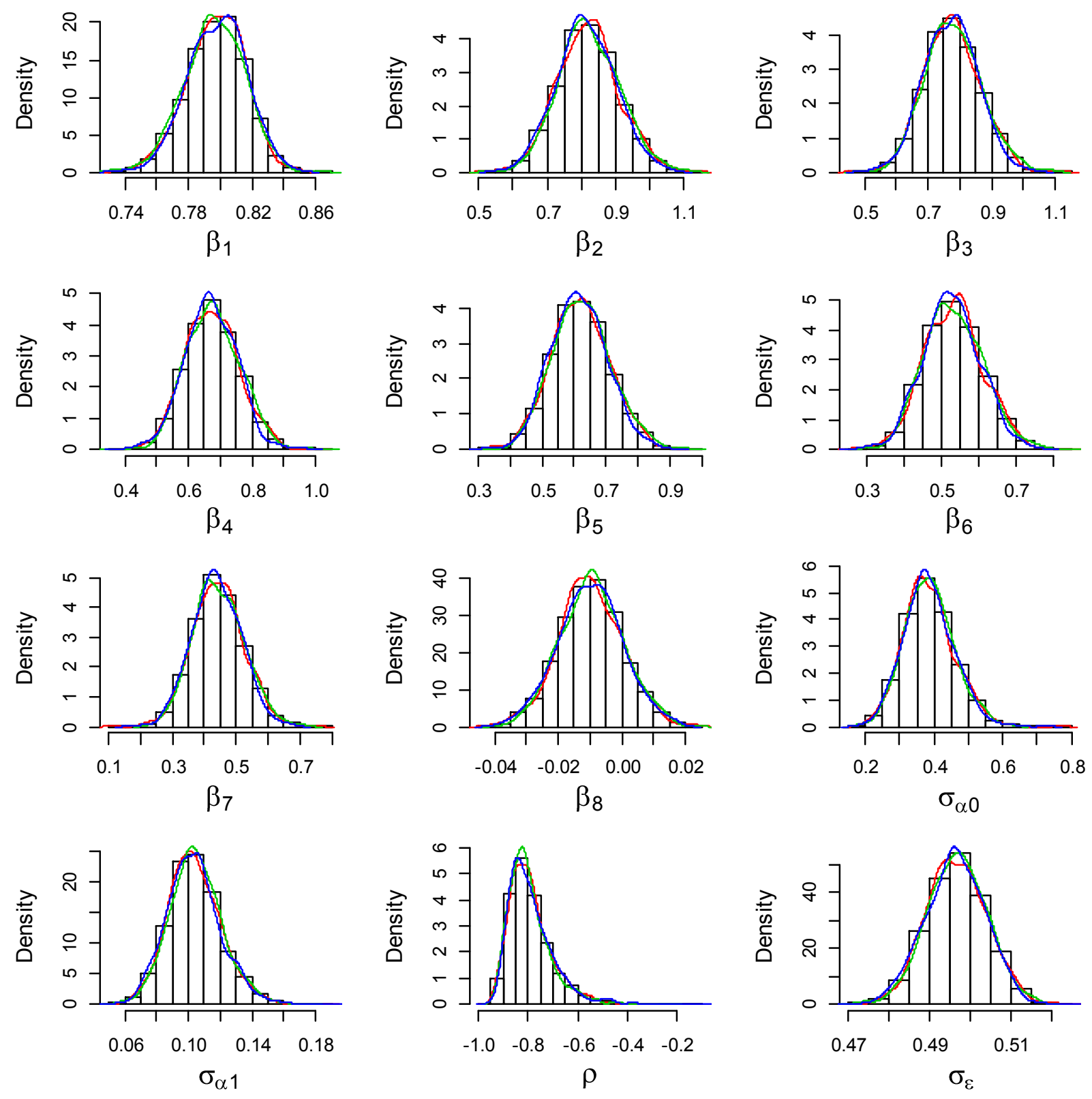

694 Fig. A3. Marginal posterior probability density distributions for parameters. Green, red and blue

695 lines show three independent MCMC chains with different initial values. The histogram is the 696 total sample of all three chains. See Eq. 1 and Table 1 for definitions of parameters. 
699 Appendix 3. BUGS code

700 BUGS.model <- function() \{

701 for (i in 1:n) \{

$702 \quad \mathrm{y}[\mathrm{i}] \sim \operatorname{dnorm}(\mathrm{y} \cdot \mathrm{hat}[\mathrm{i}]$, tau.model)

$703 \quad y . h a t[i]<-a 0[o b s[i]]+a 1[o b s[i]] * x[i]+b . s p[s p[i]] \quad$ \# model 2

$704 \quad \#$ y.hat $[i]<-$ aO $[$ obs $[i]]+a 1\left[o b s[i] *^{*} x[i]+b . s p[s p[i]]+b . b f * b f[i] \quad \#\right.$ model 4

$705\}$

706 tau.model <- pow(sigma.model,-2)

707 sigma.model $\sim$ dunif(sigma.min,sigma.max) \# prior

708 for (i in 1:6) \{b.sp[i] dunif(b.min,b.max)\} \# 6 species factor levels

709 \# b.bf dunif(b.min,b.max) \# sea state

710 for (j in 1:n.obs) \{

$711 \quad \mathrm{a} 0[\mathrm{j}]<-\mathrm{A}[\mathrm{j}, 1]$

$712 \quad a 1[j]<-A[j, 2]$

$713 \quad A[j, 1: 2] \sim \operatorname{dmnorm}($ A.hat$[j]$, Tau. $A[]$,

714 A.hat $[\mathrm{j}, 1]<-0 \quad$ \# mean of intercept random effects

715 A.hat $[\mathrm{j}, 2]<-$ b1 \# mean of slope random effects

$716\}$

717 b1 dunif(b.min,b.max) \# prior

718 Tau.A[1:2,1:2] <- inverse(Sigma.A[,])

719 Sigma.A[1,1] <- pow(sigma.a0,2)

720 Sigma.A[2,2] <- pow(sigma.a1,2)

721 Sigma.A[1,2] <- rho*sigma.a0*sigma.a1

722 Sigma.A[2,1] <- Sigma.A[1,2]

723 sigma.a0 dunif(sigma.min,sigma.max) \# prior

724 sigma.a1 dunif(sigma.min,sigma.max) \# prior

725 rho $\sim \operatorname{dunif}(-1,1)$

\# prior

$726\}$

727 
Appendix 4. R code for RJMCMC analysis

729 \# RJMCMC calibration analysis for ETP dolphin school size estimation

$730 \quad \#$

731 library(tcltk2) \# for progress bar

732 \#\# Proposal distributions for parameters for RJ step

733 rjprop.mean.sp <- rep $(0,5)$

734 rjprop.mean.bft $<-0$

735 rjprop.sd.bft <- 0.1

736 rjprop.sd.sp <- rep $(0.3,5)$

737 \#\# Proposal distributions for parameters for $\mathrm{MH}$ step

738 mhprop.sd.int <- 0.035

739 mhprop.sd.ph <- 0.007

$740 \quad$ mhprop.sd.sp $<-\operatorname{rep}(0.04,5)$

741 mhprop.sd.bft <- 0.005

742 mhprop.sd.sd.model <- 0.01 I

743 mhprop.sd.sd.obs.int $=0.01$

744 mhprop.sd.sd.obs.ph $=0.01$

745 mhprop.sd.params <- c(mhprop.sd.int, mhprop.sd.ph, mhprop.sd.sp, mhprop.sd.bft,

746 mhprop.sd.sd.model, mhprop.sd.sd.obs.int, mhprop.sd.sd.obs.ph)

747 names(mhprop.sd.params) <- c('sd.int','sd.ph',rep('sd.sp',5),'sd.bft',

748 'sd.sd.model','sd.sd.obs.int','sd.sd.obs.ph')

749

750

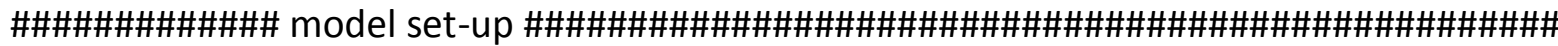

751

752

\#\# Starting values for the parameters

753 int. $0<-0.7 \quad$ \# intercept

754 ph. $0<-0.8$ \# slope for photo

755 sp. $0<-$ rjprop.mean.sp \# factor covariate with 6 levels (first level absorbed in the intercept)

756 bft.0<-rjprop.mean.bft \# beaufort coefficient

757 sd.model. $0<-0.5$ \# standard deviation of model errors

758 \# random effects for observers

759 sd.obs.int. $0<-0.2 \quad$ \# intercept for regression

760 re.obs.int <- rnorm(n.obs, 0 ,sd.obs.int.0)

761 names(re.obs.int) <- sort(unique(observers))

762 sd.obs.ph. $0<-0.05$

763 re.obs.ph <- rnorm(n.obs,0,sd.obs.ph.0)

764 names(re.obs.ph) <- sort(unique(observers))

765 params <- c(int.0,ph.0,sp.0,bft.0,sd.model.0,sd.obs.int.0,sd.obs.ph.0)

766 names(params)<-

767 c('int','ph',paste("sp",levels(species)[2:6],sep="."),'bft','sd.model','sd.obs.int','sd.obs.ph')

768 param.list <- matrix $(0,4,8)$

769 param.list $[1, \mathrm{c}(1,2)]<-1$

770 param.list $[2, \mathrm{c}(1: 7)]<-1$ 
param.list $[3, c(1,2,8)]<-1$

param.list $[4,1: 8]<-1$

\# choose the model

cur.mod <- 1

\# which parameters are switched on

\#\# Prior limits for parameters

prior.params.hi $<-1$

783 prior.sd.lo $<-0$

prior.sd.hi $<-1$

\# number of iterations, about 3000 per hour

n.iter $<-3000 * 70$ \# total number of iterations

\# setting up matrices that will store the posterior samples

$\mathrm{nr}<-$ round(n.iter/n.thin, 0$)+1 \quad$ \# number of rows is thinned no. of updates + starting value params.mat <- matrix(NA, nr,length(params)) colnames(params.mat) <- names(params) params.mat[1,] <- params colnames(re.obs.int.mat) <- paste("obs",levels(observers),".int",sep="'") re.obs.int.mat[1,]<- re.obs.int

\# vector for storing model choices

804 \# the predictor

$805 \mathrm{x}<-$ - I.photo

806 \# the response

$807 \mathrm{y}<-$ I.best

809 \#\#\#\#\#\# the likelihood equations $\log$. lik <- function $(y=y, x=x$, params = params, re.obs.int $=$ re.obs.int, re.obs. ph $=$ re.obs.ph $)\{$ sp.params<-params[3:7] \# these will be zero if beaufort is not included in the model

812 bft.params<-params[8] \# these will be zero if species is not included in the model

$813 \mathrm{mu}<-$ params['int'] + re.obs.int[observers] + (params['ph'] + re.obs.ph[observers]) * $x+$ $814 \mathrm{c}(0$, sp.params) $[$ match(species,levels(species) $)]+$ bft.params[1]*beaufort 
$815 \log$. lik <- sum(log(dnorm(y,mu,params['sd.model'])) $)+$

816 sum(log(dnorm(re.obs.ph,0,params['sd.obs.ph']))) +

817 sum(log(dnorm(re.obs.int,0,params['sd.obs.int'])), na.rm=T)

$818 \quad$ log.lik

$819\}$

820

821 \# test

$\log . \operatorname{lik}(y=$ I.best, $x=$ I.photo, params = params, re.obs.int = re.obs.int, re.obs.ph = re.obs.ph)

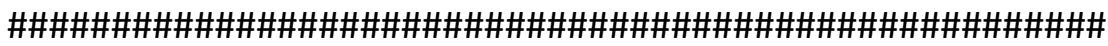

\# progress bar

$\mathrm{pb}<-$ tkProgressBar(title $=$ "progress bar", $\min =0, \max =\mathrm{n}$. iter, width $=200$ )

\# the RJMCMC algorithm

isave <- $1 \quad$ \# set the counter; first value is starting value

\#\#\#\#\# the RJ step

if(cur.p[3]==0)\{\# if species is currently not in the model, propose to add it newparams[3:7] <- rnorm(5,rjprop.mean.sp,rjprop.sd.sp) \#\#\#\#\#\#\#\#\#\#\#\#\# changed from 1 to 5 new.lik <- $\log . \operatorname{lik}(y=y, x=x$, params = newparams, re.obs.int = re.obs.int, re.obs.ph $=$ re.obs.ph) cur.lik <- $\log . \operatorname{lik}(y=y, x=x$, params = params, re.obs.int = re.obs.int, re.obs.ph = re.obs.ph) num <- new.lik + sum(log(dunif(newparams[3:7],prior.params.lo,prior.params.hi))) \# add priors for new parameters den <- cur.lik + sum(log(dnorm(newparams[3:7],rjprop.mean.sp,rjprop.sd.sp))) \# add proposal densities for new parameters

$$
A<-\min (1, \exp (\text { num-den)) }
$$

$$
\mathrm{V}<- \text { runif(1) }
$$

$$
\text { ifelse(V<=A,\{params[3:7]<-newparams[3:7]; cur.p[3:7]<-1\},\{newparams[3:7]<-params[3:7]\}) }
$$

$$
\text { else\{ \# if species is currently in the model, propose to delete it }
$$

$$
\text { newparams[3:7] <- } 0
$$

$$
\text { new.lik <- log.lik(y=y, } x=x \text {, params = newparams, re.obs.int }=\text { re.obs.int, re.obs.ph }=
$$
re.obs.ph)

cur.lik <- $\log . \operatorname{lik}(y=y, x=x$, params = params, re.obs.int = re.obs.int, re.obs.ph = re.obs.ph) num <- new.lik + sum(log(dnorm(params[3:7],rjprop.mean.sp,rjprop.sd.sp))) \# add proposal densities for current parameters

den <- cur.lik + sum(log(dunif(params[3:7],prior.params.lo,prior.params.hi))) \# add priors for current parameters

$858 \quad$ V<-runif(1) 
$860\}$

861 if(cur.p[8]==0)\{ \# if beaufort is currrently not in the model, propose to add it

862 newparams[8] <- rnorm(1,rjprop.mean.bft,rjprop.sd.bft)

863 new.lik <- $\log . \operatorname{lik}(y=y, x=x$, params = newparams, re.obs.int $=$ re.obs.int, re.obs.ph $=$

864 re.obs.ph)

865 cur.lik <- log.lik $(y=y, x=x$, params $=$ params, re.obs.int $=$ re.obs.int, re.obs.ph $=$ re.obs.ph)

num <- new.lik + sum(log(dunif(newparams[8],prior.params.lo,prior.params.hi))) \# add priors for new parameters den <- cur.lik + sum(log(dnorm(newparams[8],rjprop.mean.sp,rjprop.sd.sp))) \# add proposal densities for new parameters $A<-\min (1, \exp ($ num-den)) $\mathrm{V}<-$ runif(1) ifelse $(\mathrm{V}<=\mathrm{A},\{$ params $[8]<-$ newparams[8]; cur.p $[8]<-1\},\{$ newparams[8]<-params[8]\}) \} else\{ \# if beaufort is currently in the model, propose to delete it newparams[8] <- 0 new.lik <- $\log . \operatorname{lik}(y=y, x=x$, params = newparams, re.obs.int $=$ re.obs.int, re.obs.ph $=$ re.obs.ph) densities for current parameters

den <- cur.lik + sum(log(dunif(params[8],prior.params.lo,prior.params.hi))) \# add priors for current parameters

884 V<-runif(1)

885 ifelse $(\mathrm{V}<=\mathrm{A},\{$ params$[8]<-$ newparams[8];cur.p[8]<-0\},\{newparams[8]<-params[8]\})

$886\}$

$887 \quad$ \# which model did we end up with?

888 cur.mod<-match(sum(cur.p),apply(param.list,1,sum))

\#\#\#\#\# the $\mathrm{MH}$ step

891 newparams <- params

new.re.obs.int <- re.obs.int

893 new.re.obs.ph <- re.obs.ph

894 \# updating the parameters

895 \# the first level of species coefficients or beaufort coefficients are always zero, don't need

896 updating

897 for ( $p$ in which(cur. $p==1))$; \# paramters which can be negative

$898 \mathrm{u}<-\operatorname{rnorm}(1$, params[p], mhprop.sd.params[p])

899 newparams[p] <- u

900 new.lik <- log.lik $(y=y, x=x$, params = newparams, re.obs.int $=$ re.obs.int, re.obs.ph $=$

901 re.obs.ph)

902 cur.lik $<-\log . \operatorname{lik}(y=y, x=x$, params $=$ params, re.obs.int $=$ re.obs.int, re.obs.ph $=$ re.obs.ph $)$ 
num <- new.lik + log(dunif(newparams[p], prior.params.lo,prior.params.hi))

904 den <- cur.lik + $\log$ (dunif( params[p],prior.params.lo,prior.params.hi))

$905 \quad A<-\min (1, \exp ($ num-den))

$906 \quad$ V<-runif(1)

907 ifelse(V<=A,params[p]<-newparams[p],newparams[p]<-params[p])

$908\}$

909 for ( $p$ in 9:11) \{ \# st dev cannot be negative

$910 \mathrm{u}<-\operatorname{rnorm}(1, \operatorname{params}[\mathrm{p}]$, mhprop.sd.params[p])

911 newparams[p] $<-\mathrm{u}$

912 new.lik $<-\log . \operatorname{lik}(y=y, x=x$, params = newparams, re.obs.int $=$ re.obs.int, re.obs.ph $=$

913 re.obs.ph)

914 cur.lik $<-\log . \operatorname{lik}(y=y, x=x$, params = params, re.obs.int $=$ re.obs.int, re.obs.ph $=$ re.obs.ph)

915 num <- new.lik + $\log$ (dunif(newparams[p], prior.sd.lo,prior.sd.hi))

916 den <- cur.lik + log(dunif( params[p],prior.sd.lo,prior.sd.hi))

$917 \quad A<-\min (1, \exp ($ num-den))

$918 \quad$ V<-runif(1)

919 ifelse $(\mathrm{V}<=A$,params $[\mathrm{p}]<-$ newparams$[\mathrm{p}]$, newparams $[\mathrm{p}]<-\operatorname{params}[\mathrm{p}])$

$920\}$

921

922

923

924

925

926

940 \# each "n.thin-th" iteration, store the parameter values in matrices

941 if $(\mathrm{b} \% \%$ n.thin < 1$)\{$

942 isave $<-$ isave +1

943 params.mat[isave,] <- params

944 re.obs.int.mat[isave,] <- re.obs.int

945 re.obs.ph.mat[isave,] <- re.obs.ph

946 model[isave] <- cur.mod 
$947\}$

$948 \quad$ \# display progress

949 Sys.sleep(1)

950 setTkProgressBar(pb, b, label=paste(round(b/n.iter*100),"\% completed",sep="'"))

$951\} \quad$ \#\#\# end of iteration loop

952 close(pb); date()

953

954 \#\#\#\#\#\#\#\#\#\#\#\#\#\#\# end of RJMCMC sampling

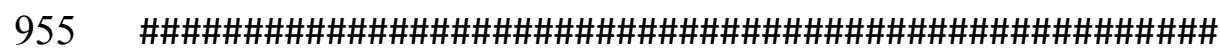

956 
958 Appendix 5. Coverage of predicted group sizes

959 For each group size estimate for each observer, we predicted group size using Eq. 1 and 960 sampling the MCMC chains from model 2 as described in Methods. For each of the 2,435

961 observer estimates, we determined if the $95 \%$ credibility interval of predicted size included the

962 photo count (our measure of true group size). Coverage of the $95 \%$ interval, measured as the

963 fraction of intervals which included the photo count, was 0.955 . We note that this procedure was

964 an inverse prediction - that is, although the model fitted $y$ to $x$, we predicted $x$ given $y$. We also

965 note that this procedure was not cross-validation, since the model was not refit for each of the

9662,435 observer estimates. Therefore, since the value being predicted (photo count) was included

967 in the model fitting, coverage was expected to be positively biased. Given the large sample size,

968 however, we believe the positive bias due to the inclusion of a single datum would be small, as

969 indeed it seemed to be. Fig A4 shows observer estimates and posterior distributions of predicted

970 group size plotted against photo count for a selection of the 59 observers. 


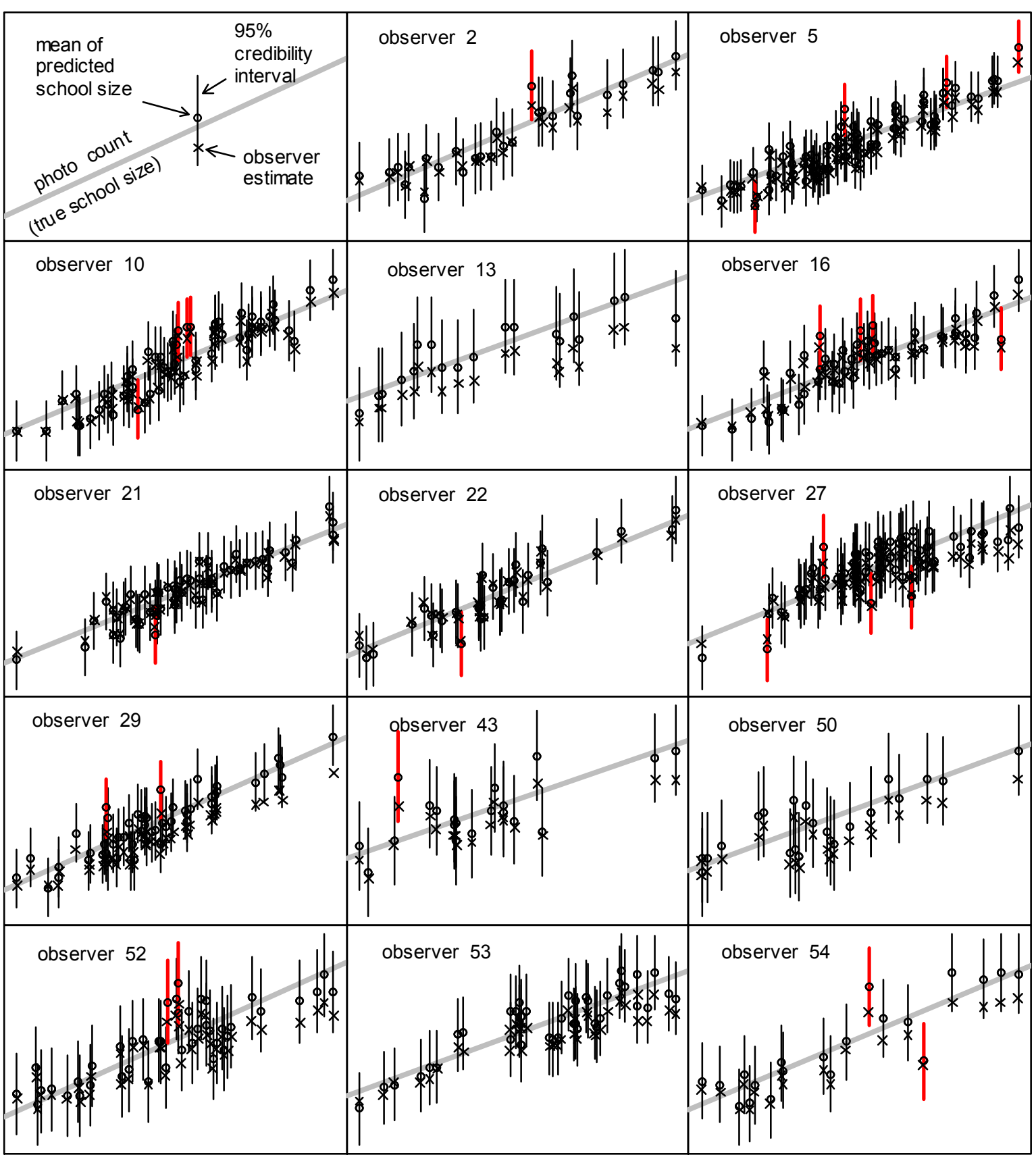

973 Fig. A4. Observer estimates (x), and group sizes predicted from those estimates, plotted relative

974 to photo count (gray line) for selected observers. Circles are the means and vertical line

975 segments the $95 \%$ credibility intervals of predicted group sizes. Cases for which the $95 \%$

976 credibility interval did not include the photo count are shown in red. 